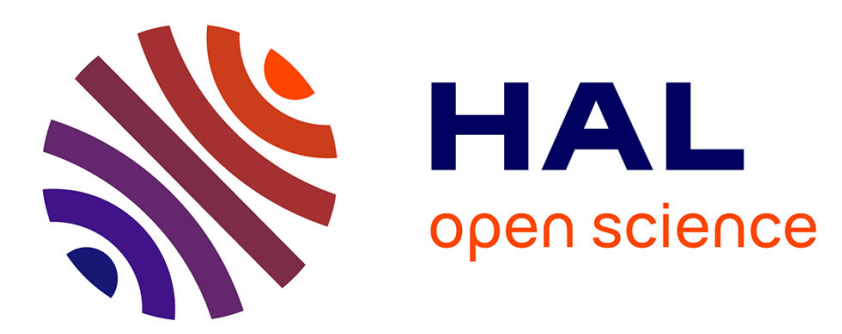

\title{
Maximum network lifetime with optimal power/rate and routing trade-off for Wireless Multimedia Sensor Networks
}

\author{
Nesrine Khernane, Jean Couchot, Ahmed Mostefaoui
}

\section{- To cite this version: \\ Nesrine Khernane, Jean Couchot, Ahmed Mostefaoui. Maximum network lifetime with optimal power/rate and routing trade-off for Wireless Multimedia Sensor Networks. Computer Communi- cations, 2018, 124, pp.1 - 16. hal-02182832}

\section{HAL Id: hal-02182832 \\ https://hal.science/hal-02182832}

Submitted on 13 Jul 2019

HAL is a multi-disciplinary open access archive for the deposit and dissemination of scientific research documents, whether they are published or not. The documents may come from teaching and research institutions in France or abroad, or from public or private research centers.
L'archive ouverte pluridisciplinaire HAL, est destinée au dépôt et à la diffusion de documents scientifiques de niveau recherche, publiés ou non, émanant des établissements d'enseignement et de recherche français ou étrangers, des laboratoires publics ou privés. 


\title{
Maximum Network Lifetime with Optimal Power/Rate and Routing trade-off for Wireless Multimedia sensor Networks
}

\author{
Nesrine Khernane ${ }^{\mathrm{a}}$, Jean-François Couchot ${ }^{\mathrm{a}}$, Ahmed Mostefaoui ${ }^{\mathrm{a}}$ \\ ${ }^{a}$ FEMTO-ST Institute Disc dep, University of Bourgogne Franche Comte, Belfort 9000, France
}

\begin{abstract}
Wireless Multimedia Sensor Networks (WMSN) are today considered as a promising technology, notably because of the availability of miniaturized multimedia hardware (e.g., CMOS cameras). Nevertheless, they do raise new research challenges; i.e., multimedia content is much more voluminous and rich in comparison to scalar one. Hence, multimedia data need pre-processing, namely coding, before they are delivered to the sink. In WMSN, meeting the required visual quality at the sink whilst taking into account the intrinsic network limitations, energy in particular, remains very challenging. In fact, higher visual quality of the data increases its volume, which in turn consumes more network resources and vice versa. The problem is more complex when including the data routing, especially when the routing is initially unknown. In this paper, we focus on the problem of simultaneously optimizing the video encoding at the source nodes and the routing of the generated data (i.e., the routing matrix is initially unknown) to the sink in order to maximize the network lifetime. This issue is tackled through the proposition of an analytic model. Based on the latter, two solutions have been studied. In the first one, of a static nature (i.e., network topology is static), the routes are calculated using the shortest path routing protocol toward the sink. The evaluation we made has shown that our proposed solution consumes less than $0.030 \%$ of the total battery while it increases the whole network lifetime nearly between 7 to 12 times, depending on the considered topologies, in comparison to the baseline approach (i.e., without optimization). Afterwards, in order to handle dynamic topology changes and to consider the link reliability, the work have been extended to the selection of routing paths, in a fully distributed fashion (i.e., using a local decision hop-by-hop routing protocol) with respect to both the shortest ones and their reliability. The second solution was evaluated through two different dynamic topologies. The simulation results show an energy consumption inferior to $0.025 \%$ and $0.080 \%$ of the total battery in the first and the second topology, respectively, with an improvement of the network lifetime that is multiplied by around 7 in comparison to the baseline approach.
\end{abstract}

Keywords: WMSNs, Video quality, Routing, Network lifetime, Distributed algorithm, Encoding power, Source rate, Reliability

\section{Introduction}

Unlike traditional wireless sensor network (WSN), wireless multimedia sensor networks capture rich multimedia content (video, images, etc.). Multimedia sensor networks, mean in that case a collection of nodes that are able to acquire multimedia data (video, images, audio) in addition to scalar measurements (temperature, humidity, etc.), to process them and finally to deliver results, through multi-hop communications, to a central node, called sink. Upon receiving this data, the sink, by means of data fusion, processing and analysis techniques, delivers to upper layers (application, end-user or cloud) relevant information. Wireless Multimedia Sensor Networks cover a large band of real applications within several domains (environmental, wild, military, industrial, etc.).

To illustrate this point, smart parking can be used as an example, among many others. In fact, recent statis-

Email addresses: nesrine.khernane@univ-fcomte.fr (Nesrine Khernane), jean-francois.couchot@univ-fcomte.fr (Jean-François Couchot), amhed.mostefaoui@univ-f comte.fr (Ahmed Mostefaoui) 
tics [1] show that on average people spend a total of nine full days per year looking for free parking spaces, especially in mega-cities which experience heavy traffic near malls, restaurants or shopping centres. This phenomenon is more pronounced in downtown areas, which lack the necessary space to build new parking. Car traffic is often synonymous with air pollution and noise, in addition to the "wasted" time experienced by drivers when they are looking/waiting for a parking box. To cope with such a problem, one can use presence sensors, buried in the ground such as SENSIT [2] (installed in Moscow, Boulevard Ring). However, the deployment of such systems requires costly roadway works. The usage of WMSNs could be an interesting alternative to such expensive systems, since they do not require costly deployments [3].

\subsection{Motivations}

In order to send the video surveillance to the cloud for relevant analyzes (such as, deep learning), the latter requires the best possible video quality, and the longest possible network lifetime.

Usually, in WVSNs, two orthogonal requirements are at odds: (a) higher video quality at the sink for analysis purposes which leads to voluminous data versus (b) preserving network resources to prolong its lifetime. In other words, WMSN should ensure the transmission of multimedia content including snapshots (i.e., images) and streaming multimedia, which fundamentally differs from traditional scalar data (i.e., high data rates, additional energy, memory and computational power requirement). Furthermore, the desired quality of the multimedia content at the sink is often the major issue that impacts the application of such networks. Thus, it is necessary to elaborate solutions in order to respect the desired video quality at the destination level whilst ensuring the maximization of the network lifetime.

Even though Multimedia content can be compressed in order to minimize data rates, it remains voluminous for WMSNs resources. Indeed, the increase of compression level reduces the number of bits to be transmitted but at the same time increases the video distortion at the destination level and conversely. Hence, two main challenges should be considered: a) the encoding of the multimedia content and $\mathbf{b}$ ) the reliable delivery of the latter. On the one hand, the encoding of the captured multimedia data is highly depending on the desired quality. Thus, finding a balance between the visual quality at the sink and the video coding at the source nodes is a crucial task. On the other hand, the reliable delivery (i.e., the chosen routing protocol) of the video content can highly influence the network's lifetime as shown in [4, 5] (several routing protocols were implemented as adds-on; each of which was considered as an input).

In fine, processing and delivering multimedia content are not independent tasks, and their interaction has a major impact on the network lifetime.

\subsection{Contributions}

Several approaches have been proposed to ensure a power/rate tradeoff for network lifetime maximization using power-rate-distortion models [6, 7]. However, the routing issue has been left as a separate issue (i.e., the routing matrix was pre-defined and considered as an input), and none of the previous research works, to the extent of our knowledge, have considered an unknown routing matrix.

In this paper, we confine our interest to the integration of the routing issue, based on an unknown routing matrix, in the analytic model proposed in our previous work [5]. In order to prolong the network lifetime, the distributed solution proposed in [5] ensures a trade-off between the desirable visual quality at the sink and the available network's resources.

Thus, a solution is proposed with the following main contributions:

- A proof that the problem of choosing a single routing protocol among $n$ routing protocols is NPcomplete,

- Based on different criteria and on an unknown routing matrix, a solution is proposed that optimally selects the forwarding nodes, based on the shortest path solution,

- Different from our previous work in [4], where the routing protocols where implemented as an inputs to the analytic model [5] (to study their impact on the network lifetime), in this paper, based on the same analytic model, we propose a solution that optimally selects the forwarding nodes.

- The resolution of the novel optimization problem is semi-distributed and can handle the topology change, in contrast to the approaches proposed in the literature.

Furthermore, two other solutions ensuring a multipath routing with disjoint paths are presented. The first solution, with two paths, from the source to the sink, can handle high rates communications and the second one includes an additional path to cope with link failure (the third link acts as an alternative path in case of 
path or link failure). An in-depth simulation analysis of the proposed approaches was conducted over two main parameters: battery consumption and network lifetime. The simulation results first show that our proposed solution, ensures the convergence of the system. Second, the latter consumes less than $0.03 \%$ of the total battery and thus ensures a prolongation of the network lifetime between 7 to 12 times, depending on the considered topologies, in comparison to the baseline approach (i.e., without optimization).

The motivation behind this paper can be described as follows: a) prolonging the network lifetime as much as possible while respecting the desirable visual quality of the data at the sink, and b) the reliable delivery of the latter. Thus, the following points can be stressed:

- The proposed solution optimally selects the forwarding nodes, based on both an energy-aware geographic forwarding and link reliability.

- Our novel solution allows an end-to-end routing with local decisions at each video sensor node without end-to-end path discovery and maintenance. In other terms, our solution is completely distributed and hence can cope with dynamic change of network topology,

- Furthermore, we propose a new dynamic path selection mechanism. The latter allows the choose of the sufficient number of paths needed to ensure a reliable data transmission and traffic distribution. The number of paths selected should respect the optimal data rate generated at each video node and links capacity.

In order to evaluate the performance of our solution, an in-depth simulation analysis of the proposed approach through two different topologies was conducted. In the first one we place the sink in the corner of the network, while in the second one, we place the sink in the middle of the network. The simulation results show that the network lifetime was increased by 7.47 and 7.67 times for the first and second topology, respectively, in comparison to baseline approach.

\subsection{Organization}

The remainder of this paper is organized as follows: Section 2 presents the related work. In Section 3 the problem of choosing a single routing protocol among $n$ routing protocols is shown to be a NP-complete. Section 4 describes the network model. Then, the problem definition and formulation, as well as the resolution of the latter is presented in section 5. Simulation results of the proposed solution for lifetime maximization under unknown traffic matrices are presented in section 6 . Section 7 targets the reliable selection of the downstream nodes with respect to the updating parameters of both: data processing and routing axes. In Section 8 the formulation and the resolution of such a problem are detailed. Simulation results of the fully distributed solution are presented in section 9 . Finally, concluding remarks and future directions are presented in section 10 .

\section{Related work}

Several approaches have been proposed in the literature to deal with routing in WMSNs. However, it was targeted as a separate issue without considering the energy consumption at each node for data encoding. On the other hand, the power-rate distortion solutions, only focus on how to find and ensure an optimum power-rate resource allocation, while assuming a pre-defined routing matrix. In this section some of the proposed solutions in the literature are outlined, which can be broadly classified into two categories.

\subsection{Power-Rate-Distortion approaches}

Based on the Power-rate-Distortion (P-R-D) model [6], He et al. [8] have proposed a fully decentralized solution to maximize the network lifetime in WMSN. A mathematical model that jointly considers the source rates, the encoding powers, and the data delivery distortion was developed and solved through the Lagrangian duality and Subgradient method. However, the proposed model does not ensure the convexity at any initial configuration which may lead to problems with no feasible solution.

Using the same P-R-D model, authors in [9] aim to minimize the power consumption, provide adaptive processing for video encoding while maintaining the QoS required by the application. To do so, an efficient solution based on the Lagrange duality is developed and the relaxation mechanism is used to avoid solving the integer nonlinear problem.

Through another P-R-D model, proposed in [7], authors in [10] have proposed a distributed quality-lifetime maximization solution in WMSN, where they integrate the distortion model in the objective function instead considering it as a constraint. The problem was formulated based on Generalized Network Utility Maximization (GNUM) [11], and solved by using the Proximal Point Algorithm [12]. However, the inverse lifetime was computed in a centralized manner by the sink and sent back to sources. 
Similarly, an appropriate trade-off between the minimum data distortion and maximum network lifetime was studied in [13]. The formulated problem was solved in a distributed manner by primal decomposition. In the same context, authors [14] have proposed a solution that jointly optimizes the network coding based multi-path routing. A fully distributed algorithm was presented and solved through the Lagrange and Subgradient method. However, both of the aforementioned solutions used the network coding during problem formulation. The latter requires the decoding and the encoding of the transmitted data at the intermediate nodes resulting in an additional power consumption.

The problem formulated in [15] takes into account not only the processing distortion (i.e., P-R-D [6]) but also the channel distortion model. The objective function aims at minimizing the energy consumption of each node in the network that combines both static video nodes and cluster heads (i.e., clustered topology). Then, the problem was solved using the proximal minimization algorithm [12]. However, authors did not consider the reception power at the cluster head while formulating the objective function. They did not mention if they used the one-hop or multi-hop mechanism, since if the multi-hop mechanism is used the flow conservation at each video or cluster head should be considered. On the other hand, if the one-hop is used this means that the cluster heads should be as close as possible to the sink. In addition to that, the proposed solution is centralised, the capacity of each link was fixed, and the retransmission mechanism was not considered.

In the context of Ultra-Dense Networks (UDNs), authors in [16] have proposed a solution that mainly combines video coding (using scale video coding (SVC) and fountain coding (FC) methods), content updates (using proactive and active update strategies), and video scheduling in which the number of participating users should not be larger than three. However, this proactive content update strategy cannot be applied in wireless video sensor networks due to the hard resource constraints of sensor nodes.

In the same context, authors in [17] addressed the problem of device-to-device (D2D) media content sharing. They proposed an efficient algorithm in order to match the content demanders with the potential provider. To ensure a high physical link quality, they introduced the social-aware rate at which the devices should communicate. However, the energy consumption of such a solution may be too large, and thus can not be brought directly in wireless video sensor networks.

Furthermore, all of the aforementioned approaches especially focus on the optimal power-rate-distortion trade-off, and do not consider the routing issue that has been left as a separate field (i.e., the routing matrix was pre-defined and considered as an input in the optimization process).

\subsection{Routing approaches}

Authors in [18] have shown that the problem of routing messages in a wireless sensor network so as to maximize network lifetime is NP-hard. As a consequence, they propose a heuristic solution. The latter is based on delaying as much as possible the depletion of a sensors' energy to a level below that needed to transmit to its closest neighbor, while in [19] authors have focused on the problem of computing a flow that maximizes the lifetime of the network. The problem is formulated as a linear programming problem and sub-gradient algorithms are used to solve it in a distributed manner. However, in both [18] and [19] the transmission rate on each link is fixed and only payload transmission power was considered. Whereas, the encoding power was not considered.

Authors in [20] have proposed a framework to obtain an optimal tradeoff between communication and computation power consumption leading to network lifetime maximization under the delay quality of service constraint. The distributed implementation of the algorithm realizing the proposed framework is achieved using duality theory. The convexity of the optimization problem was solved differently compared to our solution and the problem was divided into two sub-problems.

In order to meet delays, packet-loss, energy and memory requirements, authors in [21] have only addressed the routing scheme between the cluster heads and the sink. However, each sensor node periodically broadcasts a HELLO packet and forwards ants that have to reach the sink and go back for route discovery. Thus, control packets used in this solution for route discovery can cause a high overhead.

By exploiting the geographic locations and QoS performance of the neighbor nodes, authors in [22] have implemented a localized hop-by-hop routing. Moreover, the protocol (almost) ensures a homogeneous energy dissipation rate for all routing nodes in the network through a multi-objective Lexicographic Optimizationbased geographic forwarding. The problem was formulated using the multi-objective Lexicographic Optimization (LO) approach. Moreover, LO exploits only local information to make routing decisions. The absence of a global routing scheme reduces the networks setup and updating costs.

In [23], authors addressed the mesh topology, in which the E-Mesh route information collector and E-Mesh 
route selection should be maintained at each node. Based on the E-mesh route information collector, that contains the remaining energy, current load, and node position, the E-mesh route selection calculates the utility.

Nonetheless, the aforementioned routing protocols did not consider at all the encoding power at each node, which can affect not only the desired video quality, but also the network lifetime.

\subsection{Routing Versus Power-Rate-Distortion approaches}

To the best of our knowledge, the only work that considered both: the power-rate-distortion optimization, and the routing discovery in WMSNs, for network lifetime maximization is described in [24]. The proposed algorithm selects a set of paths, that join every active node to the sink. However, the optimization problem does not consider the reception power consumption that has a direct impact on the network lifetime. Additionally, the routing and channel allocation information are monitored by the sink (i.e., in a centralized manner), and must be updated at nodes.

\section{The problem}

Including the routing issue in our optimization problem leads to the following question: what is the best way to select, among all available ones, the optimal routing path ? The "brute-force" approach consists in testing all the existing routing paths and then selecting the optimal one with regard to the lifetime metric. However, the number of possible routing paths can be very huge and testing all of them consumes time and energy. That is way was privileged an heuristic approach.

Let $\mathcal{P}_{1}$ be the problem of executing $N$ routing protocols on a single instance of a network. Each routing protocol has an execution time denoted $t_{i}$. The main objective here is to find a scheduling that minimizes the positive number $L=\sum_{i=1}^{N} t_{i}$. This function tends to minimize the execution time of the routing protocols.

Theorem 1. The decision problem corresponding to the aforementioned problem is NP-complete.

Proof 1. Let $N$ be the number of routing protocols, with $t_{i}$ the execution time of the routing protocol $i$, and a positive number $T$. Is there a scheduling such as $L \leq T$ ?

- Given a solution, it is clear that the time taken to verify whether it is valid or not is $O(n)$.
- The problem, denoted by $\mathcal{P}_{2}$, that will be reduced to $\mathcal{P}_{1}$ is the Knapsack problem, which is defined as follows:

A set $S=\left\{x_{1}, x_{2}, x_{3}, \ldots, x_{n}\right\}$ of $n$ numbers and a positive number $y$ such that: $\sum_{i=1}^{n} x_{i}=y$, is there $A \subset S$ such that: $\sum_{i=1}^{n} x_{i} \leq y$ ?

Let us now construct the polynomial reduction $f$ of the Knapsack problem to our $\mathcal{P}_{1}$ problem, in such a way that an instance $I$ of the Knapsack problem, has a "yes" answer, if and only if $f(I)$, an instance of our problem, has a "yes" answer. Let $I$ be an instance of Knapsack problem. An instance $f(I)$ of our problem can be formulated as follows:

- $N=n+1$

- $t_{i}=x_{i} ; \sum_{i=1}^{N} t_{i}=\sum_{i=1}^{n} x_{i}+1$;

- $t_{n+1}=1 ; \sum_{i=1}^{n+1} t_{i}=y+1$.

We will prove now that $L \leq T$ if and only if there is a set $A$ such that $\sum_{i=1}^{n} x_{i} \leq y$. Suppose that the subset $A$ such that $\sum_{i=1}^{n} x_{i} \leq y$ exists. Then, consider a scheduling that executes first the subset $A$ in an arbitrary order, then the $n+1$ routing protocol, afterward the $S-A$ subset. The routing protocols in a subset $A$ end at $y$ in the worst cases, since $\sum_{i=1}^{n} x_{i} \leq y$. The $y$ routing protocol ends at $n+1$, and the rest of the routing protocols end at $\sum_{i=1}^{n} x_{i}+1$. Thus all routing protocols were executed and thus $L \leq T$.

It can concluded that if the Knapsack problem has a solution then $\mathcal{P}_{1}$ also has a solution.

Suppose now that our problem $\mathcal{P}_{1}$ has a solution, thus it has to be proven that the Knapsack problem also has a solution. In order to prove this implication, it is simpler to show its contraposition. In other words, suppose that the Knapsack problem does not have a solution which implies that there is no solution $A \subset S$ such that $\sum_{i=1}^{n} x_{i} \leq y$. Let $A$ be the subset of the routing protocols executed before the $n+1$ routing protocol, which implies that the execution of $A$ ends at: $E_{A}=\sum_{i=1}^{n} x_{i}>y$, by hypothesis. The $n+1$ routing protocol will be executed after and ends at $E_{A}+1$, and thus $L>T$.

Therefore, if the Knapsack problem has a negative response, then so does $\mathcal{P}_{1}$. The Knapsack problem is equivalent to our problem, and thus, it can be deduced that $\mathcal{P}_{1}$ is $\mathrm{Np}$-complete.

\section{Network Model}

Let us consider a multimedia sensor network consisting of $\mathcal{N}$ identical stationary multimedia sensors. Each of 
which knows its coordinates, by means of any localization mechanism [25, 26], and the coordinate of the sink. Each node $h$ has a communication range denoted ComRange, and generates a multimedia traffic of rate $R_{h}$ to be sent to the sink.

Upon the network deployment, the neighboring table is constructed by the means of a Hello message broadcasted by each node. Using this step, each node can also record the coordinates of its immediate neighbors (used later on for geographic routing).

\subsection{Geographic progression}

As a first step to our approaches, we considered the geographic progression of the generated data flow from source nodes toward the sink. This step allows to assign an orientation to each link in the network. In other works, this orientation follows the data traffic flow. The motivation behind doing so is to "simplify" the data routing on the one hand and on the other hand to follow the video data flow which is voluminous. Figure 1 shows an example of a directed WMSNs composed of 9 sensor nodes and one sink deployed in a region of $50 \mathrm{~m} \times 50 \mathrm{~m}$. The orientation of each link has been set following the geographic progression to the sink.

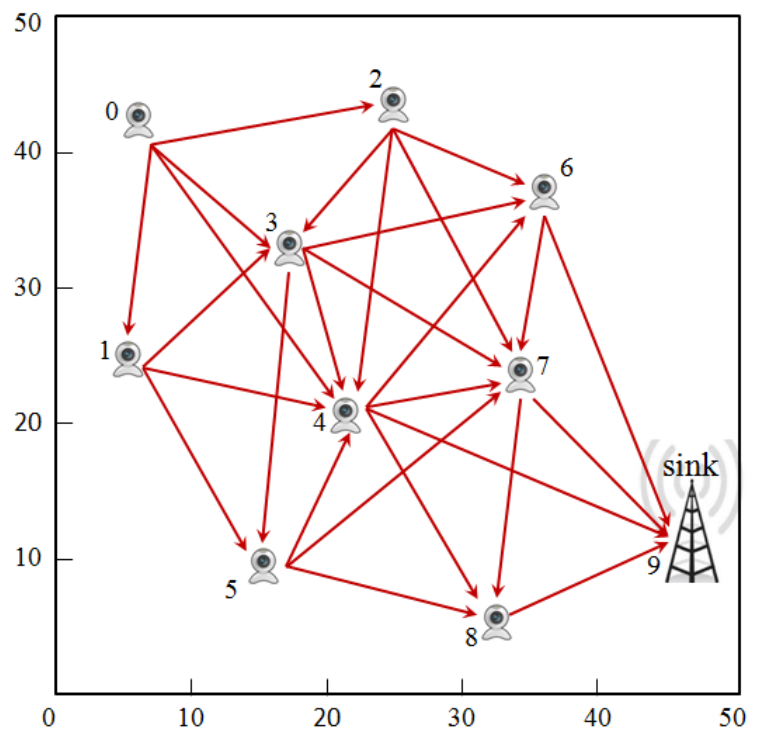

Figure 1: Example of a WMSN.

Formally, the network can be defined as follows:

- An oriented graph $G(\mathcal{V}, L)$, where $\mathcal{V}=$ $\left\{h_{1}, \ldots, h_{n}\right\}$ is a set of video sensor nodes and $\mathcal{L}=\left\{l_{i j} \mid h_{i}, h_{j} \in \mathcal{V}\right\}$ is a set of oriented links.
- Two routing matrices $a_{i l}^{+}$and $a_{i l}^{-}$of size $\mathcal{N} * \mathcal{L}$ denote the matrices of outgoing links and incoming links, respectively. Where the ith row represents the ith path, while the lth column represents the lth link, whose elements are defined as: $a_{i l}^{+}$(resp. with $a_{i l}^{-}$matrix) equals to 1 , if a given link $l$ is an outgoing link from $i$ (resp. with an incoming link) and 0 otherwise. The construction of $a_{i l}^{+}$and $a_{i l}^{-}$is based on the distance to the sink. More formally, if the distance of $j$ (neighbor of $i$ through the link $l$ ) to the sink is less than the distance of $i$ to the sink, then, the $a_{i l}^{+}=1$ and $a_{j l}^{-}=1$.

- From the $a_{i l}^{+}$and $a_{i l}^{-}$matrices, a general routing matrix $a_{i l}$ of size $\mathcal{N} * \mathcal{L}$ can be constructed as the following: $a_{i l}=a_{i l}^{+}-a_{i l}^{-}$.

The generated traffic $R_{h}$ can be forwarded directly to the sink, if the latter is in the communication range of node $h$. Otherwise, node $h$ needs to use a multi-hop communication. In other words, it forwards the generated traffic $R_{h}$ to its one hop neighboring nodes that will proceed similarly until reaching the sink node. Thus, each intermediate node $i$ that contributes to the data routing of node $h$ needs to use its own memory. Therefore, the flow conservation [8], denoted $\eta_{h i}$, at each node can be formulated as follows:

$\eta_{h i}=\sum_{l \in \mathcal{L}} a_{i l} x_{h l}= \begin{cases}R_{h} & \text { if } i \text { is the source of the traffic } \\ -R_{h} & \text { if } i \text { is the sink } \\ 0 & \text { otherwise. }\end{cases}$

where $x_{h l}$ represents the data rate, originated from node $h$, at link $l$.

\subsection{Node Power Consumption Model}

As previously mentioned, the interaction between the processing and the delivery of multimedia content has a major impact on the network lifetime. Thus, the video coding and routing are complex tasks that consume energy and hence impact the overall energy of the network. In this subsection, the different powers consumed by each node for both video coding and multimedia data transport are developed.

\subsubsection{Video coding power consumption}

The main objective here is to consider and minimize as much as possible the video distortion, since the distortion level is highly influenced by the video coding. Authors in [8, 6] have proposed the following power-ratedistortion (P-R-D) analytical model:

$$
D_{h}=\sigma^{2} e^{-\gamma \cdot R_{h} \cdot P_{s h}^{2 / 3}}
$$


where $\sigma^{2}$ is the average input variance, $D_{h}$ is the encoding distortion, and $\gamma$ is the encoding efficiency coefficient, $R_{h}$ is the source rate and $P_{s h}$ is the encoding power. From this model, it can be deduced that when the encoding power $P_{s h}$ is too high or too low, the distortion level will become large. Thus, an optimal encoding power should be determined and can be derived from (2) as the following:

$$
P_{s h}=\left(\frac{\ln \left(\sigma^{2}\right)-\ln \left(D_{h}\right)}{\gamma * R_{h}}\right)^{\frac{2}{3}}
$$

\subsubsection{Transmission power consumption}

Using the power Consumption model presented in [27], the data transmission from node $i$ is formulated as:

$$
P_{t i}=\sum_{l \in \mathcal{L}} a_{i l}^{+} *\left(\alpha+\beta d_{l}^{n_{p}}\right) * \sum_{h \in \mathcal{V}} x_{h l}
$$

where $\alpha$ and $\beta$ are transmit electronics parameters, $d_{l}$ is the distance between the transmitter and the receiver, $n_{p}$ is the path-loss exponent [28] and $\sum_{h \in \mathcal{V}} x_{h l}$ corresponds to the aggregate rate transmitted through link $l$.

\subsubsection{Reception power consumption}

Using the same power Consumption model, the required power needed for data reception at node $i$ can be formulated as:

$$
P_{r i}=c^{r} * \sum_{l \in \mathcal{L}} a_{i l}^{-} * \sum_{h \in \mathcal{V}} x_{h l}
$$

where $c^{r}$ is the radio receiver energy consumption cost. Thus, the total power dissipation at node $i$ can be formulated as:

$$
P_{i}=P_{s h}+P_{t i}+P_{r i}
$$

where $P_{s h}=0$, if $i$ is the sink node $(i . e ., i=\mathcal{N})$.

\subsection{Network Power Consumption Model}

In this paper, we consider critical applications where the exhaustion of the energy of the first node will cause the failure of the whole network. Therefore, and by assuming that each node $i$ has initial energy denoted $B_{i}$, the network lifetime $T_{n e t}$ is defined as:

$$
T_{n e t}=\min _{i \in \mathcal{N}} B_{i} / P_{i}
$$

\subsection{Distributed Bellman Ford formulation}

In this subsection the aim is to minimize as much as possible the energy consumed by each node for data transmission. Based on the literature, the shortest path (i.e., paths) represents the minimum-energy routing topology if data are not aggregated [29, 30]. For this reason, the distributed Bellman Ford algorithm was implemented with a slight adaptations that will be explained in the next section.

The distributed Bellman Ford approach has the following formulation [31]:

$$
\mathcal{D}_{i}=\min _{j}\left[d_{i j}+\mathcal{D}_{j}\right]
$$

where $D_{i}$ is the shortest distance from node $i$ to the destination and $d_{i j}$ is the distance from node $i$ to $j$ ( $j$ is a one-hop neighbor of $i$, denoted by $\mathrm{Nbrs}_{i}$ ). The inclusion of the minimum in (8) means that the best neighbor is selected to be included to the list of the shortest paths from $i$ to the destination.

\subsection{Disjoint Paths}

The benefits of disjoint path routing are significant for high data multimedia applications [32]. It can be used to split the high data over the existing paths or even to cope with the links' failure. However, the disjoint paths problem is known to be NP-complete in directed graphs even if the number of disjoint paths is equal to 2 . In this paper, two routing protocols are proposed with 2 and 3 paths, respectively, based on the Bellman Ford method. The problem of the disjoint paths [33] can be defined as follows:

Given a directed network $G=(\mathcal{N}, \mathcal{L})$ of $\mathcal{N}$ nodes and $\mathcal{L}$ weighted links. Find $k$ paths $p_{1}, p_{2}, \ldots, p_{k}$ from $i \in \mathcal{N}$ to the sink node, such that the paths share minimal common links (or nodes).

\section{Power/Rate and Routing Optimization}

\subsection{Problem formulation}

As previously mentioned, we aim to integrate the routing problem into the analytic model proposed in [5] that ensures a trade-off between the desirable visual quality at the sink and the available network's resources.

Before going further, let us briefly recall the analytical model proposed in our previous work [5].

The maximization of the network lifetime can be expressed by minimizing the inverse lifetime given in (7). Let $q=1 / T_{\text {net }}$ be the inverse lifetime of the network. Thus, the problem can be formulated as follows:

$$
\begin{aligned}
& \underset{\left(q, r, x, P_{s}\right)}{\operatorname{minimize}} \mathrm{q} \\
& \text { subject to } \sum_{l \in \mathcal{L}} a_{i l} x_{h l}=\eta_{h i} \quad \forall h \in \mathcal{V} \forall i \in \mathcal{N}, \\
& \sigma^{2} e^{\gamma R_{h} P_{s h}^{2 / 3}} \leq D_{h} \quad \forall h \in \mathcal{V}, \\
& P_{s h}+P_{t i}+P_{r i} \leq q B_{i} \quad \forall i \in \mathcal{N}, \\
& x_{h l} \geq 0, R_{h} \geq 0, P_{s h}>0
\end{aligned}
$$


In order to solve this problem in a distributed manner, an auxiliary variable, $q_{i}(\forall i \in N)$, has been introduced and maintained at each individual node $i$ that should be followed by the following constraint: $\sum_{i \in N} a_{i l} . q_{i}=0$ $(\forall l \in \mathcal{L})$. Firstly, to ensure the convexity of the objective function, minimizing $q$ is equivalent to minimizing $|N| q^{2}$, which is equivalent to: $\sum_{i \in N} q_{i}^{2}$, using the auxiliary variable $q_{i}$. On the other hand, the following powers have been introduced to the corresponding functions (i.e., $R_{h}, x_{h l}$ and $P_{s h}$ ): 2, 2, and 8/3 respectively, with some regular factors (namely $\delta_{r}, \delta_{x}$ and $\delta_{p}$ ), to ensure a strict convexity of the problem, more details can be found in [5]. Then, the problem in (9] can be reformulated as follows:

$$
\begin{aligned}
& \underset{\left(q, r, x, P_{s}\right)}{\operatorname{minimize}} \sum_{i \in N} q_{i}^{2}+\delta_{x} \sum_{h, l} x_{h l}^{2}+\delta_{r} \sum_{h} R_{h}^{2}+\delta_{p} \sum_{h} P_{s h}^{8 / 3} \\
& \text { subject to } \sum_{l \in \mathcal{L}} a_{i l} x_{h l}=\eta_{h i} \quad \forall h \in \mathcal{V} \forall i \in \mathcal{N} \text {, } \\
& \sigma^{2} e^{-\gamma R_{h} P_{s h}^{2 / 3}} \leq D_{h} \quad \forall h \in \mathcal{V}, \\
& P_{s h}+P_{t i}+P_{r i} \leq q_{i} B_{i} \quad \forall i \in \mathcal{N}, \\
& \sum_{i \in \mathcal{N}} a_{i l} q_{i}=0 \quad \forall l \in \mathcal{L} \\
& x_{h l} \geq 0, R_{h} \geq 0, P_{s h}>0 \text {. }
\end{aligned}
$$

Let us now introduce the routing constraints to 10 :

$$
\underset{\left(q, r, x, P_{s}\right)}{\operatorname{minimize}} \sum_{i \in N} q_{i}^{2}+\delta_{x} \sum_{h, l} x_{h l}^{2}+\delta_{r} \sum_{h} R_{h}^{2}+\delta_{p} \sum_{h} P_{s h}^{8 / 3}
$$

subject to

$$
\begin{aligned}
& \text { 1) } \sum_{l \in \mathcal{L}} a_{i l} x_{h l}=\eta_{h i} \quad \forall h \in \mathcal{V} \forall i \in \mathcal{N}, \\
& \text { 2) } \sigma^{2} e^{-\gamma R_{h} P_{s h}^{2 / 3}} \leq D_{h} \quad \forall h \in \mathcal{V}, \\
& \text { 3) } P_{s h}+P_{t i}+P_{r i} \leq q_{i} B_{i} \quad \forall i \in \mathcal{N}, \\
& \text { 4) } \sum_{i \in \mathcal{N}} a_{i l} q_{i}=0 \quad \forall l \in \mathcal{L}, \\
& \text { 5) } \mathcal{D}_{i}=\min _{j}\left[d_{i j}+\mathcal{D}_{j}\right] \quad \forall i \in \mathcal{N} \forall j \in \text { Nbrs }_{i} \\
& \text { 6) } a_{i l}^{+}=\{0,1\} \quad \forall i \in \mathcal{N} \forall l \in \mathcal{L}, \\
& \text { 7) } a_{i l}^{-}=\{0,1\} \quad \forall i \in \mathcal{N} \forall l \in \mathcal{L}, \\
& \text { 8) } x_{h l} \geq 0, R_{h} \geq 0, P_{s h}>0
\end{aligned}
$$

The first constraint reflects the flow conservation law, maintained at each node. The second constraint ensures the respect of the desired video quality expected at the sink. The third constraint presents the network lifetime with respect to the minimum node lifetime. The fourth constraint ensures the convergence of the system with respect to $q_{i}$. The fifth constraint presents the shortest path from $i$ to the destination going through the best neighbor. The sixth and seventh constraints present the indicators of the sender and the receiver, respectively. Remaining constraints ensure that all the variables remain positive.

\subsection{Problem resolution}

In this section we present the resolution of Problem (11). Due to the rich structure of this problem, a decomposition approach can be applied in order to split the latter into a set of small subproblems. Thus, these latter can be solved in a distributed manner and converge to the global optimum [34]. Firstly, a primal decomposition with respect to the coupling variables $\left(a_{i l}^{+}, a_{i l}^{-}\right)$is required. Then, the dual problem can be formulated with respect to the coupling constraints (1), (2), (3) and (4). Finally, the original optimization problem (11) can be decomposed into two subproblems as follows:

$$
\begin{aligned}
& \underset{\left(q, r, x, P_{s}\right)}{\mathbf{P} 1: \min } \sum_{i \in N} q_{i}^{2}+\delta_{x} \sum_{h, l} x_{h l}^{\alpha_{x}}+\delta_{r} \sum_{h} R_{h}^{\alpha_{r}}+\delta_{p} \sum_{h} P_{s h}^{\alpha_{p}} \\
& \text { subject to }(1),(2),(3),(4),(8) . \\
& \underset{\left(a_{i l}^{+}, a_{i l}^{-}\right)}{\text {22:min }} U^{*}\left(a_{i l}^{+}, a_{i l}^{-}\right) \\
& \text {subject to (5), (6), (7). }
\end{aligned}
$$

Note that the optimization of the $\mathbf{P 1}$ problem is achieved if and only if the coupling variables $\left(a_{i l}^{+}, a_{i l}^{-}\right)$are fixed. Since, $\left(a_{i l}^{+}, a_{i l}^{-}\right)$are updated through $\mathbf{P 2}, U^{*}\left(a_{i l}^{+}, a_{i l}^{-}\right)$can be viewed as the optimal value for the $\mathbf{P 1}$ problem and used to provide an approximation to the global optimal solution.

\subsection{1. $P 2$ resolution}

Next, the way to update the coupling variable $\left(a_{i l}^{+}, a_{i l}^{-}\right)$ is discussed, based on the distributed Bellman Ford method, to solve the $\mathbf{P 2}$ problem. The path (paths) discovery steps is executed according to the following steps:

Neighboring discover. In this phase each video sensor node $i \in \mathcal{V}$ broadcasts a HELLO message in order to have the geometric coordination of its neighbors, and updates its neighboring table. At the end of this phase, each node $i$ will have the routing matrices of its outgoing and incoming links (i.e., $a_{i l}^{+}$and $a_{i l}^{-}$, respectively), as well as the routing matrices of the outgoing and incoming links of its one-hop neighbors $j \in \operatorname{Nbrs}_{i}$ (i.e., $a_{j l}^{+}$and 
Path establishment. After the neighboring discovery phase, each sensor node $i$ initiates the Bellman Ford algorithm by sending $d_{i j}+\mathcal{D}_{i}$, where $\mathcal{D}_{i}=0$ if $i$ is the source and $\mathcal{D}_{i}=\infty$ otherwise. At the reception of such a message each node $j \in N_{b r s}$ checks if $\mathcal{D}_{j}>d_{i j}+\mathcal{D}_{i}$. If it returns true $j$ proceeds as follows:

- Set $\mathcal{D}_{j}=d_{i j}+\mathcal{D}_{i}$,

- marks the sensor node $i$ as the best current predecessor,

- sends the estimate $d_{j k}+\mathcal{D}_{j}$ to each sensor node $k \in N b r s_{j}$,

- the operation continues until the sink node.

Path confirmation. At the end of the path establishment step, the sink node should maintain the shortest distance from each node to the latter as well as the latest node through which it has received this distance. Then, in the path confirmation phase the sink node sends a pathConf message through the inverse path.

Disjoint Path. In order to construct multi-paths, the sink node instead of maintaining only the shortest distance from each node to the latter, maintains the two or three shortest distances paths.

Let us recall that the intermediate nodes have to send not only their own data, but also the data of their incoming neighbors which has a direct impact on their energy consumption and hence on the network lifetime. Thus, the main goal behind the use of disjoint paths is to balance the network load over the intermediate nodes. In other terms, the generated data from node $h$ will be divided on the existing outgoing neighbors. Now, let us suppose that we will not ensure the disjoint paths, figure 2 can expose one of the undesired cases:

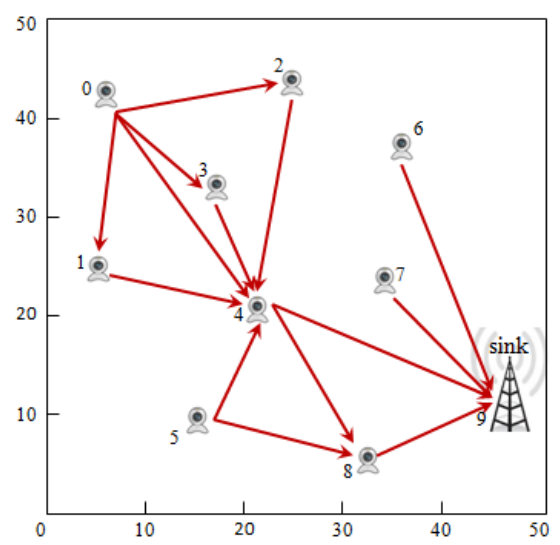

Figure 2: Network with shared node
Our solution is fully distributed and each node only has the vision of its one hop neighboring. From figure 2 , node 0 sends its own traffic to its neighboring nodes assuming that it has 4 paths, except that all the traffic will end up in node 4 . This type of situation can rapidly bring down the energy of the intermediate node and affect the whole network lifetime.

Thus, to ensure a disjoint paths and avoid to have paths with a shared node, each node is limited into accepting only one message from an intermediate node with a given sequence number. Thus, node that receives more than one message, maintains the one with the minimum cost and ignores the rest.

\subsubsection{P1 resolution}

Lagrangian Dual based methods [34] can be used to solve such a problem. Thus, the Lagrangian function for the optimization problem P1 can be written as follows:

$$
\begin{aligned}
& L\left(R, x, P_{s}, q, u, v, \lambda, w\right)= \\
& \sum_{i \in \mathcal{N}} q_{i}^{2}+\delta_{x} \sum_{h, l} x_{h l}^{\alpha_{x}}+\delta_{r} \sum_{h} R_{h}^{\alpha_{r}}+\delta_{p} \sum_{h} P_{s h}^{\alpha_{p}}+ \\
& \sum_{h \in \mathcal{V}} \sum_{i \in \mathcal{N}} u_{h i}\left(\sum_{l \in \mathcal{L}} a_{i l} \cdot x_{h l}-\eta_{h i}\right) \\
& +\sum_{h \in \mathcal{V}} v_{h}\left(\frac{\ln \left(\sigma^{2} / D_{h}\right)}{\gamma P_{s h}^{2 / 3}}-R_{h}\right) \\
& +\sum_{i \in \mathcal{N}} \lambda_{i}\left(P_{i}-q_{i} B_{i}\right) \\
& +\sum_{l \in \mathcal{L}} w_{l}\left(\sum_{i \in \mathcal{N}} a_{i l} q_{i}\right)
\end{aligned}
$$

For any $h \in \mathcal{V}, i \in \mathcal{N}$, and $l \in \mathcal{L}$, and where $u_{i}, v_{h}$, $\lambda_{h, i}, w_{l}$ are the Lagrange multipliers associated with the different constraints of the optimization problem.

Following the subgradient method [35], the different Lagrange multipliers can be iteratively calculated, as follows:

$$
\begin{aligned}
& u_{h i}^{k+1}=u_{h i}^{k}-\theta^{k}\left(\eta_{h i}^{k}-\sum_{l \in \mathcal{L}} a_{i l} \cdot x_{h l}^{k}\right) \\
& v_{h}^{k+1}=\max \left\{0, v_{h}^{k}-\theta^{k}\left(R_{h}^{k}-\frac{\ln \left(\sigma^{2} / D_{h}\right)}{\gamma\left(P_{s h}^{k}\right)^{2 / 3}}\right)\right\} \\
& \lambda_{i}^{k+1}=\max \left\{0, \lambda_{i}^{k}-\theta^{k}\left(q_{i}^{k} B_{i}-P_{t i}^{k}-P_{r i}^{k}\right)\right\} \\
& w_{l}^{k+1}=w_{l}^{k}+\theta^{k} \sum_{i \in \mathcal{N}} a_{i l} q_{i}^{k}
\end{aligned}
$$

Where $\theta^{k}$ represents the step size and given by: $\theta^{k}=$ $\rho / k^{1 / 2}$, where $\rho>0$ and $k>0$.

As motioned in our previous work [5], all the functions to be minimized are differentiable, and thus, can be computed as follows: 


$$
\begin{aligned}
& P_{s h}^{k}= \\
& \max \left\{\epsilon,\left(\frac{-3 \lambda_{h}^{k}+\sqrt{\left(3 \lambda_{h}^{k}\right)^{2}+64 \delta_{p} \cdot v_{h}^{k} / \gamma \cdot \ln \left(\sigma^{2} / D_{h}\right)}}{16 \delta_{p}}\right)^{\frac{3}{5}}\right\} \\
& R_{h}^{k}=\max \left\{0, \frac{v_{h}{ }^{k}}{2 \delta_{r}}\right\} \\
& X_{h, l}^{k}=\max \left\{0, \frac{-\sum_{i \in \mathcal{N}}\left(\lambda_{i}^{k}\left(P_{t i}+P_{r i}\right)+u_{h i}^{k} a_{i l}\right)}{2 \delta_{x}}\right\}
\end{aligned}
$$

for any $h \in \mathcal{V}, i \in \mathcal{N}$, and $l \in \mathcal{L}$.

\section{Simulation results}

In this section, our routing/power-rate tradeoff approach is evaluated, through simulation. The model was implemented using OMNET++ simulator [36], through MiXiM framework [37]. The values of the simulation parameters are presented in Table 1. Different visual sensors were studied CITRIC [38], Panoptes [39] and Meerkats [40]. Among them, the choice fell on the visual sensor settings of the Panoptes taking into account the similarities between the services provided by these latter and the target monitoring and surveillance visual application requirements.

\subsection{Convergence of the proposed solution}

The convergence of the auxiliary variable $q_{i}$ to a common $q$, can be observed in figure 3 , where after a $10^{-2}$ convergence threshold, the system is seen to be almost stable. Thus, in the following, the system is considered as completely stable when the maximum variation between the $q_{i}$ is $T=10^{-2}$.

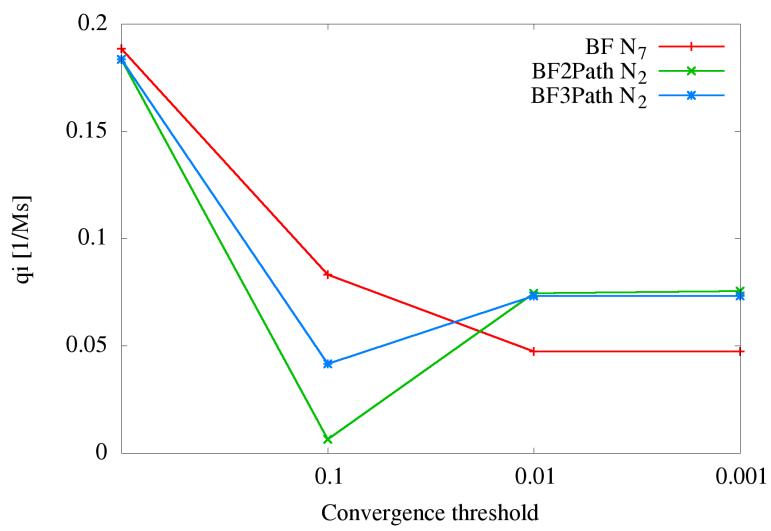

Figure 3: Auxiliary variables

For the $\mathbf{P 2}$ optimization, Table 2 shows the activity duration in seconds to find the optimal value of the coupling variables.

\begin{tabular}{|l|l|}
\hline Routing protocol & Time (s) \\
\hline Bellman Ford one path & 5.35 \\
\hline Bellman Ford two paths & 6.59 \\
\hline Bellman Ford three paths & 7.19 \\
\hline
\end{tabular}

Table 2: Activity duration of the $\mathbf{P 2}$ optimization

\subsection{Optimization cost}

In this subsection the cost of the optimization steps is analyzed, as both energy and duration at each node are considered.

\subsubsection{Energy cost}

Figure 4 depicts the percentage of battery consumption of sensor nodes which consume the most, namely, node 7 , node 2 and node 2 for one path, two paths and three paths, respectively. Thus, it concerns the total energy requirement for the optimization steps of both P1 and P2. it can be observed that the routing protocol with only two paths (BF2PATH $N_{2}$ ) is the least energy applicant routing protocol. This observation can be explained by the fact that the one path routing protocol $\left(\mathrm{BF} N_{7}\right)$ requires much more iterations to converge and thus, it can get to the point of consuming more energy. Despite this, we can conclude that the optimization steps including both $\mathbf{P 1}$ and $\mathbf{P 2}$ consume a negligible amount of energy.

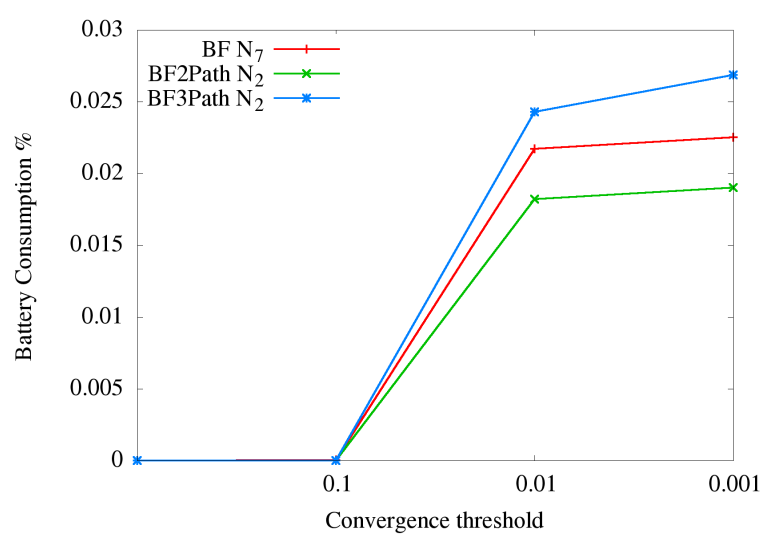

Figure 4: battery consumption of optimization steps

\subsubsection{Optimization steps duration}

At this stage of evaluation, we present the need in term of duration (in minutes) of the optimization steps to converge to the common variable $q$. Figure 5 presents the optimization steps duration including both: P1 plus P2, for each threshold $T \leq 10^{-3}$ of the last converging nodes (namely, node 7 , node 2 and node 2 for one path, two paths and three, respectively). It can be observed that, 


\begin{tabular}{|l|l|l|}
\hline Notation & Description & Value \\
\hline$\sigma^{2}$ & Variance of video encoder (in terms of MSE) & 3500 \\
\hline$\gamma$ & Encoding efficiency coefficient & $55.54 \mathrm{~W}^{3 / 2} \cdot \mathrm{Mb}^{-1} \cdot \mathrm{s}^{-1}$ \\
\hline$B_{i}$ & Initial energy at each nodes & $5.0 \mathrm{MJ}$ \\
\hline$\delta_{x}, \delta_{r}, \delta_{p}$ & Regularization factors & 0.2 \\
\hline$\rho$ & step size parameter & 0.15 \\
\hline$D_{h}$ & Distortion of an encoding frame (in terms of MSE) & 100 \\
\hline$\alpha$ & Energy cost of the transmit electronics & $0.5 \mathrm{~J} . \mathrm{Mb}^{-1}$ \\
\hline$\beta$ & Coefficient term of the transmit amplifier & $1.3 * 10^{-8} \mathrm{~J} . \mathrm{Mb}^{-1} \cdot \mathrm{m}^{-4}$ \\
\hline$n_{p}$ & Path loss exponent & 4 \\
\hline$c^{r}$ & Energy consumption cost of radio receiver & $0.5 \mathrm{~J} . \mathrm{Mb}^{-1}$ \\
\hline
\end{tabular}

Table 1: Configuration of model parameters in a WVSN [8]

if more precision is required (i.e., $T \geq 10^{-3}$, which depends on the demand of a given application), the system would need more time to be considered as completely functional (i.e., all nodes converge to a common variable $q$ ).

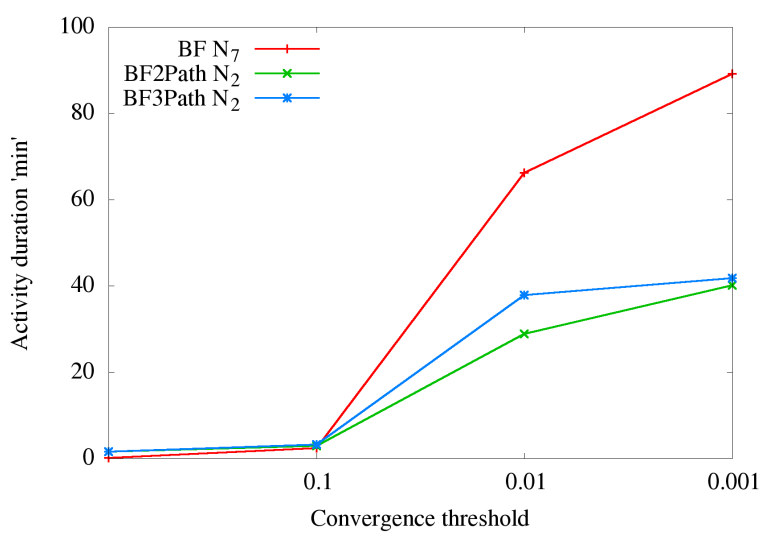

Figure 5: Optimization duration

\subsection{Node lifetime improvement}

The node lifetime can be calculated using the following formula: $T_{i}=B_{i} / P_{i}$.

Figure 6 shows the lowest improvement of nodes lifetime. it can be observed that with our optimization steps, and by including the routing constraints in the analytical model while imposing a trade-off between $P_{s h}$ and $R_{h}$, the network lifetime has been increased by at least 7.72, 12.53 and 11.95 times considering the minimum nodes lifetime (i.e., node 7 , node 2 and node 2 for one path, two paths and three, respectively). It can also be observed that routing protocols with two paths $\left(\mathrm{BF} 2 \mathrm{PATH} \mathrm{N}_{2}\right)$ and three paths $\left(\mathrm{BF} 3 \mathrm{PATH} N_{2}\right)$ ensure more network lifetime, since the multimedia content is distributed over the existing outgoing links. And thus, it demonstrates that the proposed design effectively increases the nodes lifetime and thus the network lifetime.

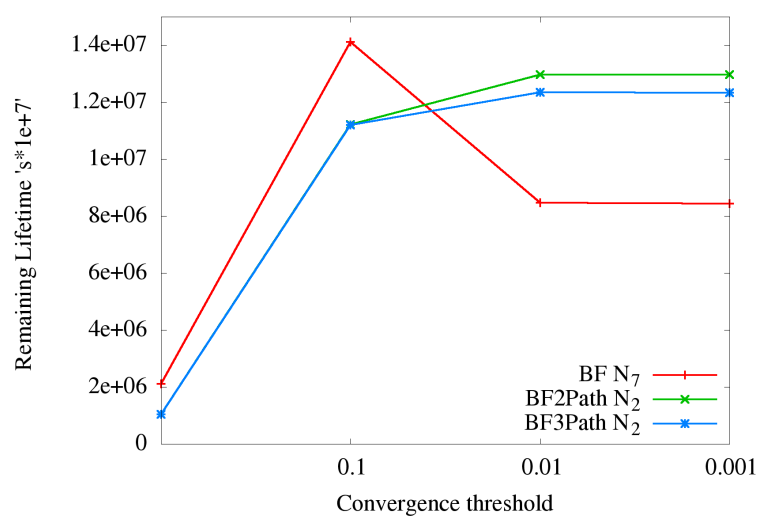

Figure 6: Remaining lifetime after each convergence threshold

\section{Maximum Network Lifetime with reliability and Energy Aware Routing}

It should be noted that the main problems in wireless multimedia sensor networks have been treated and separated mainly into two axes: a) the data processing axis and b) the routing axis. In the data processing axis the main objective is the optimal resource allocation (namely. the determination of the encoding power at the source level, and the video quality at the destination level). On the other hand, the main objective of the second axis (namely, the routing axis) is to find the optimal end-to-end path. However, treating each axis separately means that each axis ignores the parameters of the second one, which can lead to inefficient solutions.

To cope with this problem, we have proposed in the previous experimentation an analytical model $[11$ that takes as constraints the parameters of both data processing axis and routing axis. Even if the problem was divided into two sub problems (namely, P1 and P2), P1 (i.e., data processing problem) cannot be achieved without the results of $\mathbf{P 2}$. Nevertheless, it can be observed that the resolution of the $\mathbf{P 2}$ problem (i.e., routing problem) did not consider any of the parameters of the first one. 
Therefore, in this section, we extend our study and propose a fully distributed solution that respects both the optimal resource allocation and the reliable end-to-end delivery of multimedia data content. In order to maximize the network lifetime, the routing axis cannot be achieved without the data rate and links capacity parameters, determined and updated by the data processing axis. On the other hand, the data processing axis cannot be achieved without the routing tables updated by the routing axis.

\subsection{Analytical model with multirate links}

Before going further, let us first briefly discuss the novel analytic model used in the rest of this paper. In contrast with the optimization problem 10 in section 5.1. a new model was chosen with more constraints (heavily borrowed from [41]) such as the dynamic links capacity and the limited power of both the transmission and reception processes, as shown in the following formulation:

$$
\begin{aligned}
& \underset{\left(q, R, x, P_{s}\right)}{\operatorname{minimize}} \sum_{i \in \mathcal{N}} q_{i}^{2}+\delta_{x} \sum_{h, l} x_{h l}^{2}+\delta_{r} \sum_{h} R_{h}^{2}+\delta_{p} \sum_{h} P_{s h}^{8 / 3} \\
& \text { subject to } \sum_{l \in \mathcal{L}} a_{i l} x_{h l}=\eta_{h i} \quad \forall h \in \mathcal{V} \forall i \in \mathcal{N} \text {, } \\
& \sigma^{2} e^{-\gamma R_{h} P_{s h}^{2 / 3}} \leq D_{h} \quad \forall h \in \mathcal{V}, \\
& P_{s h}+P_{t i}+P_{r i} \leq q_{i} B_{i} \quad \forall i \in \mathcal{N}, \\
& \sum_{i \in \mathcal{N}} a_{i l} q_{i}=0 \quad \forall l \in \mathcal{L}, \\
& \sum_{h \in \mathcal{V}} x_{h l} \leq W \log _{2} \sqrt{1+\frac{P_{r l}}{N_{0} W}} \quad \forall l \in \mathcal{L}, \\
& \sum_{l \in \mathcal{L}} a_{i l}^{+} P_{t_{l}} \leq P_{t_{\max }} \quad \forall i \in \mathcal{N} \\
& \sum_{l \in \mathcal{L}} a_{i l}^{-} P_{r_{l}} \leq P_{r_{\max }} \quad \forall i \in \mathcal{N}, \\
& x_{h l} \geq 0, R_{h} \geq 0, P_{s h}>0, q_{i}>0,
\end{aligned}
$$

where, the Fifth constraint ensures that each data rate sent on each link respects the maximum link capacity. The Sixth and Seventh constraints ensure the respect of the maximum transmission and reception power, respectively, by each node.

\subsection{Distributed energy aware routing}

As previously mentioned, each video node knows its own coordinates as well as those of the sink node, while the coordinates of its one hop neighbors are obtained through the execution of a Hello protocol. In order to minimize as much as possible the energy consumption at each node, and to ensure a fully distributed solution needed during the scale up of the network, we consider a hop-by-hop routing, rather than the traditional end-toend path discovery routing and maintenance. The latter gives the ability for a video source node to control precisely a route so as to optimize a particular service goal (e.g., reliability, bandwidth, energy consumption). The proposed solution exploits the geographic locations to make a local decision at each video sensor node. The latter ensures a selection of the best downstream node(s) to be included to the list of the shortest path toward the sink node.

Let us give an example of such a selection: we consider the topology presented in figure 1 . For node 5 the best neighboring node that ensures the shortest path to the sink is node 8 . However, the latter may not respect the reliability requirement (for instance, the reliability of the link from 5 to 8 may be too small compared to the application reliability requirement). Thus, it could not be chosen. Therefore, node 5 chooses its second best neighboring node (i.e., node 7) that ensures both: the second shortest path to the sink and respects the reliability requirement.

The main reasons behind such a choice are: a) the possibility of scalability to large scale video sensor networks, b) the maximization of the network lifetime and c) the possibility to handle with dynamic topologies. Thus, in solution to our problem, each node $i \in \mathcal{N}$ selects its downstream node(s) using the following formula:

$$
\begin{aligned}
& \operatorname{dist}(i, \mathcal{S})= \\
& \min _{j \in N b r s_{i}}[\operatorname{dist}(i, j)+\operatorname{dist}(j, \mathcal{S}) \mid \operatorname{dist}(i, \mathcal{S}) \geq \operatorname{dist}(j, \mathcal{S})]
\end{aligned}
$$

where $\mathcal{S}$ is the sink node, and $\operatorname{dist}(i, j)$ presents the Euclidean distance from node $i$ to node $j$. Firstly, this formulation (16) ensures that the chosen node $j$, $j \in N b r s_{i}$, gives a geographic progress toward the sink node $(\operatorname{dist}(i, \mathcal{S}) \geq \operatorname{dist}(j, \mathcal{S}))$. Secondly, it ensures that the best downstream node is chosen (according to: $\min _{j \in N b r s_{i}}[\operatorname{dist}(i, j)+\operatorname{dist}(j, \mathcal{S}))$ and to be included in the list of the shortest path toward the sink.

Unlike the Bellman Ford formulation (8) presented in section 4.4. where the sink decides about the routes that each video node should take, here each node chooses its best downstream node based on its local information, and those of its one hop neighboring node (s).

\subsection{Link reliability}

Due to the hard characteristics of the wireless medium (such as: the interferences, the link quality 
degradation, the link failure, or even the dynamic network topology, the node mobility, and the congestion), reliable multimedia content transmission in wireless video sensor networks is a challenging task. Thus, selecting the most appropriate downstream nodes toward the sink node is the main objective in this paper.

Reliability is defined as the ratio of the successfully received packets $(S u c R x)$ by the destination at the link $l$, to the number of packets generated and transmitted $(T x)$ by source node at the same link $l$. Thus, for a given link $l \in \mathcal{L}$, the reliability $\mathcal{R}_{l}$ can be computed as follows:

$$
\mathcal{R}_{l}=\frac{a_{j l}^{-} S u c R x_{j}}{a_{i l}^{+} T x_{i}} \quad \forall j \in N b r s .
$$

The latter should respect the link reliability defined by the application layer, denoted RequiredReliability ${ }_{l}$ in the rest of this paper, as follows:

$$
\mathcal{R}_{l} \geq \text { RequiredReliability } \forall l \in \mathcal{L} \text {. }
$$

\subsection{Traffic distribution}

In our proposed solution, the path(s) is selected based on both the energy consumed in the data transmission process and the reliability required by the application layer. After the efficient selection of the intermediate node(s), another important issue that should be treated is the adequate number of paths that should be considered in order to achieve the performance demands of the target application. Therefore, we propose an additional path selection mechanism to choose a sufficient number of paths to provide reliable data transmission and traffic distribution.

The proposed mechanism is based on the traffic generated at each video sensor node $R_{h}, \forall h \in \mathcal{V}$, and the capacity of the channel $C_{l}$ at each link $l$ (Shannon's theorem (1984) [42]), and can be achieved as follows:

If $R_{h} \leq \sum_{l \in \mathcal{L}} a_{i l}^{+} C_{l}, \forall i \in \mathcal{N} \forall h \in \mathcal{V}$, then the number of the selected path(s) is sufficient. Otherwise, another path should be selected based on (16) and (18). Where

$$
C_{l}=W \log _{2} \sqrt{1+\frac{P_{r l}}{N_{0} W}} \quad \forall l \in \mathcal{L},
$$

Once a set of paths is selected, the protocol distributes the network traffic over the selected paths with respect to the link capacity $C_{l}$.

\section{Problem formulation and resolution}

In order to consider the parameters of both axes, the aim is to integrate the routing problem into the analytic model presented in (15). Thus, the final optimization problem can be formulated as the following:

$$
\begin{aligned}
& \underset{\left(q, R, x, P_{s}\right)}{\operatorname{minimize}} \sum_{i \in \mathcal{N}} q_{i}^{2}+\delta_{x} \sum_{h, l} x_{h l}^{2}+\delta_{r} \sum_{h} R_{h}^{2}+\delta_{p} \sum_{h} P_{s h}^{8 / 3} \\
& \text { subject to: 1) } \sum_{l \in \mathcal{L}} a_{i l} x_{h l}=\eta_{h i} \quad \forall h \in \mathcal{V} \forall i \in \mathcal{N} \text {, } \\
& \text { 2) } \sigma^{2} e^{-\gamma R_{h} P_{s h}^{2 / 3}} \leq D_{h} \quad \forall h \in \mathcal{V} \text {, } \\
& \text { 3) } P_{s h}+P_{t i}+P_{r i} \leq q_{i} B_{i} \quad \forall i \in \mathcal{N} \text {, } \\
& \text { 4) } \sum_{i \in \mathcal{N}} a_{i l} q_{i}=0 \quad \forall l \in \mathcal{L} \text {, } \\
& \text { 5) } \sum_{h \in \mathcal{V}} x_{h l} \leq W \log _{2} \sqrt{1+\frac{P_{r l}}{N_{0} W}} \quad \forall l \in \mathcal{L}, \\
& \text { 6) } \sum_{l \in \mathcal{L}} a_{i l}^{+} P_{t_{l}} \leq P_{t_{\max }} \quad \forall i \in \mathcal{N} \text {, } \\
& \text { 7) } \sum_{l \in \mathcal{L}} a_{i l}^{-} P_{r_{l}} \leq P_{r_{\max }} \quad \forall i \in \mathcal{N} \text {, } \\
& \text { 8) } \operatorname{dist}(i, \mathcal{S})=\min _{j \in N b r s_{i}}[\operatorname{dist}(i, j)+\operatorname{dist}(j, \mathcal{S}) \\
& \mid \operatorname{dist}(i, \mathcal{S}) \geq \operatorname{dist}(j, \mathcal{S})] \quad \forall i \in \mathcal{N}, \\
& \text { 9) } \mathcal{R}_{l} \geq \text { RequiredReliability }, \quad \forall l \in \mathcal{L} \text {, } \\
& \text { 10) } R h \geq \sum_{l \in \mathcal{L}} a_{i l}^{+} C_{l} \quad \forall h \in \mathcal{V}, \forall i \in \mathcal{N} \\
& \text { 11) } a_{i l}^{+}=\{0,1\} \quad \forall i \in \mathcal{N} \forall l \in \mathcal{L} \text {, } \\
& \text { 12) } a_{i l}^{-}=\{0,1\} \quad \forall i \in \mathcal{N} \forall l \in \mathcal{L} \text {, } \\
& \text { 13) } x_{h l} \geq 0, R_{h} \geq 0, P_{s h}>0, q_{i}>0 \text {. }
\end{aligned}
$$

Due to the rich structure of this problem 20 , and similarly to the problem presented in (11), a decomposition approach can also be applied to the latter. Accordingly, the original optimization problem (20) can be decomposed into two subproblems, as follows:

$$
\underset{\left(q, R, x, P_{s}\right)}{\text { P3: } \sum_{i \in N}} \sum_{i} q_{i}^{2}+\delta_{x} \sum_{h, l} x_{h l}^{2}+\delta_{r} \sum_{h} R_{h}^{2}+\delta_{p} \sum_{h} P_{s h}^{8 / 3}
$$

subject to (1), (2), (3), (4), (5), (6), (7), (13).

$$
\begin{aligned}
& \underset{\left(a_{i l}^{+}, a_{i l}^{-}\right)}{\text {P4:min }} U^{*}\left(a_{i l}^{+}, a_{i l}^{-}\right) \\
& \text {subject to (8), (9), (10), (11), (12). }
\end{aligned}
$$

Let us recall that the $U^{*}\left(a_{i l}^{+}, a_{i l}^{-}\right)$can be viewed as the optimal value for the $\mathbf{P 3}$ problem, and used to resolve the latter. 


\section{1. $P 3$ resolution}

In addition to the iterative calculation of the Lagrange multipliers described in section (5.2.2), and using the subgradient method [35], the Lagrange multipliers, i.e., $\Gamma, \mathrm{Z}$ and $\zeta$, added to the Fifth, Sixth and Seventh constraints, respectively, can be calculated as follows:

$$
\begin{aligned}
\Gamma_{l}^{k+1} & = \\
\max & \left\{0, \Gamma_{l}^{k}-\theta^{k}\left(W \log _{2} \sqrt{1+\frac{P_{r l}^{k}}{N_{0} W}}-\sum_{h \in \mathcal{V}} x_{h l}^{k}\right)\right\}, \\
\mathrm{Z}_{i}^{k+1} & =\max \left\{0, Z_{i}^{k}-\theta^{k}\left(P_{t_{\max }}-\sum_{l \in \mathcal{L}} a_{i l}^{+} P_{t l}^{k}\right)\right\}, \\
\zeta_{i}^{k+1} & =\max \left\{0, \zeta_{i}^{k}-\theta^{k}\left(P_{r_{\max }}-\sum_{l \in \mathcal{L}} a_{i l}^{-} P_{r l}^{k}\right)\right\} .
\end{aligned}
$$

Finally the primal variables $\left(q_{i}^{k}, P_{s h}^{k}\right.$ and $\left.R_{h}\right)$ can be calculated as described in section 5.2.2 to obtain a fully distributed solution. However, the calculation of the $x_{h l}^{k}$ primal variable is more complicated, since the latter appears inside the logarithm when applying the Shannon's formula. From [41] the latter can be calculated as follows:

$$
\begin{aligned}
& x_{h l}^{k}= \\
& \max \left\{0, \frac{-1}{2 \delta_{x}}\left(\sum_{i \in \mathcal{N}} u_{h i}^{k} a_{i l}+c_{l}^{s} \sum_{i \in \mathcal{N}} \lambda_{i}^{k} a_{i l}^{+}+c^{r} \sum_{i \in \mathcal{N}} \lambda_{i}^{k} a_{i l}^{-}\right.\right. \\
& \left.\left.+\Gamma_{l}^{k}+c_{l}^{s} \sum_{i \in \mathcal{N}} Z_{i}^{k} a_{i l}^{+}+c^{r} \sum_{i \in \mathcal{N}} \zeta_{i}^{k} a_{i l}^{-}-\frac{c^{r} \Gamma_{l}^{k}}{2 N_{0} \ln 2}\right)\right\} .
\end{aligned}
$$

The distributed optimization Algorithm 11 summarizes the resolution of the $\mathbf{P 3}$ optimization problem

\section{2. $P 4$ resolution}

Let us now discuss the updating of the coupling variable $\left(a_{i l}^{+}, a_{i l}^{-}\right)$, based on the localized hop-by-hop routing protocol.

Each node $i$ executes first the distributed optimization algorithm P4 described in Algorithm 2, that has as an input the required reliability (RequiredReliability,$l \in \mathcal{L}$ ) defined by the application layer, and starts by identifying all the neighboring nodes (step1, lines 3-9). In this step, called the neighboring discovery phase, a node $j$ is considered as a neighbor of $i$ if the distance between the two nodes is inferior or equal to the defined communication range (ComRange). Once the neighboring nodes are discovered, the estimation of the links reliability starts by executing a simple ping processus (step2, lines 10-13), using the formulation 18

Achieving this point, each node $i$ has the necessary information in order to select the first best downstream node. To do so, the node $i$ identifies a candidate downstream node $j\left(j \in N_{b r s_{i}}\right)$ that produces the geographic progress toward the sink node $(\mathcal{S})$ (i.e., constraint (8)), ensures a shortest path (i.e., constraint (9)) and respects at the same time the required reliability by the application layer (i.e., constraint (10)). Once this conditions are met, it stores it in the routing table, updates the $a_{i l}^{+}$and $a_{i l}^{-}$matrices and informs all its neighboring nodes (step3, lines 14-21).

Let us know explain the main part of our algorithm that allows to cross the two axes, namely, the data processing axis and the routing axis. After the update of the $a_{i l}^{+}$and $a_{i l}^{-}$matrices, each node $i$ can indeed starts the execution of the distributed optimization algorithm $\mathbf{P 3}$ described in Algorithm 1, in order to update the processing axis parameters (i.e., $P_{s h}, R_{h}, x_{h l}, q_{i}$ ) until a certain number of iterations (ItThreeshold). Once this threshold is reached, the Distributed optimization algorithm P4 (Algorithm 2-step4 line 25) is called with an updated $R_{h}$ and $C_{l}$.

Then, each node $i$ verifies if $R h \geq \sum_{l \in \mathcal{L}} a_{i l}^{+} C_{l}, \forall i \in \mathcal{N}$. If it returns True, the node $i$ executes step 3 in order to select the second best downstream node. Then recalls the distributed optimization algorithm P3 with the updated $a_{i l}^{+}, a_{i l}^{-}$and ItThreeshold as an input.

Note that this steps are repeated until $R h \leq \sum_{l \in \mathcal{L}} a_{i l}^{+} C_{l}$, $\forall i \in \mathcal{N}$.

\subsubsection{Path reliability}

The end-to-end path reliability is difficult to address, and can be defined by the application layer. Since the latter is multiplicative, the variation of only one link over one path can highly reduce the end-to-end reliability performance. Taken as an example, the end-to-end reliability requirement of $85 \%$ and only 4 hops to reach the destination from the source node. Note that, even if the reliability of each of the 4 links is of $85 \%$ then the path is not feasible to satisfy the end-to-end reliability requirement. To achieve a such reliability, the mean of reliability on the 4 links has to be $97 \%$, which is very restrictive in wireless multimedia sensor networks. Thus, this point should be considered during the link reliability definition.

\subsubsection{Ping process}

In order to estimate the reliability of each constructed link, each video sensor node $i$ sends a certain number of packets to each neighboring node $j$ attached with a sequence number. At the reception of these packets, each node $j\left(\forall j \in \mathrm{Nbrs}_{i}\right.$ ) computes the $\mathcal{R}_{l}, \forall l \in \mathcal{L}$, as described in (18). Then, it sends back the estimated reliability of the link to the source node $i$. 


\subsubsection{Path confirmation}

In contrast to the solution proposed in section 5.2.1 where each node receives the list of the adequate downstream nodes from the sink through the inverse path, in this solution, each node relies on local information to make the decision. In fact, based on the links reliability and on the updating of the outgoing and incoming links(through the updating of the $R_{h}$ and $C_{l}$ ), each node selects and maintains the adequate number of paths in order to achieve the optimal routes between the latter and the sink.

\subsubsection{Disjoint Path}

Based on the data rate $R_{h}$ at the source node $h$ and the links capacity $C_{l}$ on all the outgoing links $l$ from node $h$, each node decides locally about the sufficient number of paths that should be considered. If more than one path is selected, then the source node $h$ chooses the second node $j, j \in \mathrm{Nbrs}_{h}$, that is totally different from the first best neighbor node, and that ensures not only a geographic progress toward the sink, but also respects the required reliability by the application layer.

\section{Simulation results}

In this stage of simulation, we use the same parameters initialization values of the video sensors as presented in section 1. Then, we set the transmission and reception maximal powers to $P_{t_{\max }}=2.63 \mathrm{~W}$ and $P_{r_{\max }}=1.5 \mathrm{~W}$, respectively. The additive white Gaussian noise is set to $N_{0}=0.5$, while the bandwith is set to $W=240 \mathrm{Ghz}$. In addition to these parameters, and in order to evaluate the performance of our solution, we have chosen to evaluate it through two different topologies as shown in figure 7, where the sink was placed in two different locations. In the first topology, the sink was placed in the corner of the network, while in the second topology, the sink was placed in the middle of the network.

\subsection{Convergence of the proposed solution}

The convergence of the auxiliary variable $q_{i}$ to a common $q$, can be observed in the Figure 8 and Figure 9 for the first (T1) and second (T2) topologies, respectively. Similarly to the convergence results in section 6.1, we consider the system as completely stable when the maximum variation between the $q_{i}$ is $T=10^{-2}$.

The peaks in figures 8 and 9 can also be observed. These peaks mean that, the correspondent node has chosen to add an additional path after the data rate $R_{h}$ and links capacity $C_{l}$ verification, in order to choose the adequate number of paths to be used. Note that the duration of each peak is very small and even negligible and is about $2.17 \mathrm{~s}$ using the MiXiM-2.3 framework that was integrated into the omnetpp-4.6 simulator.

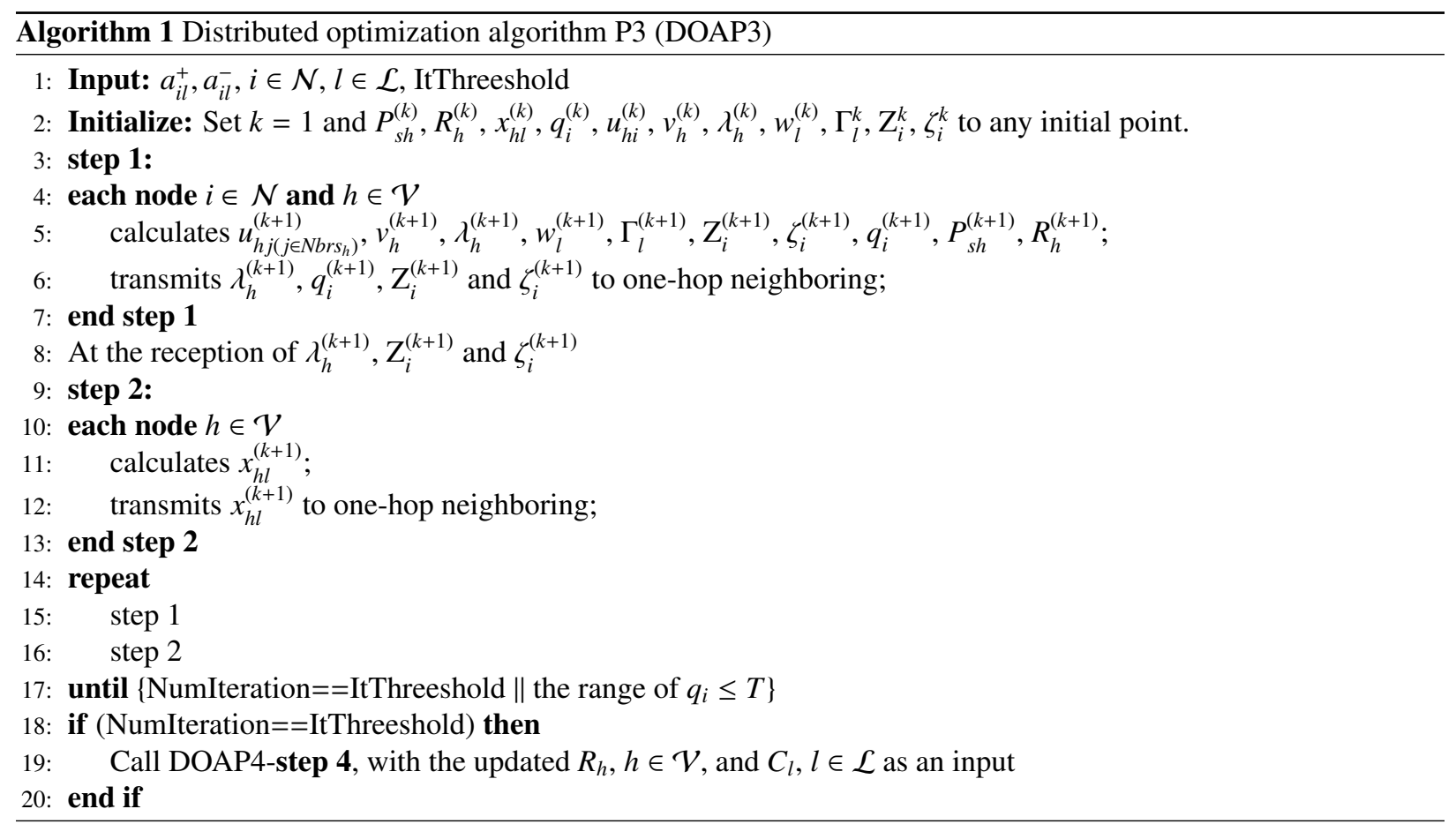




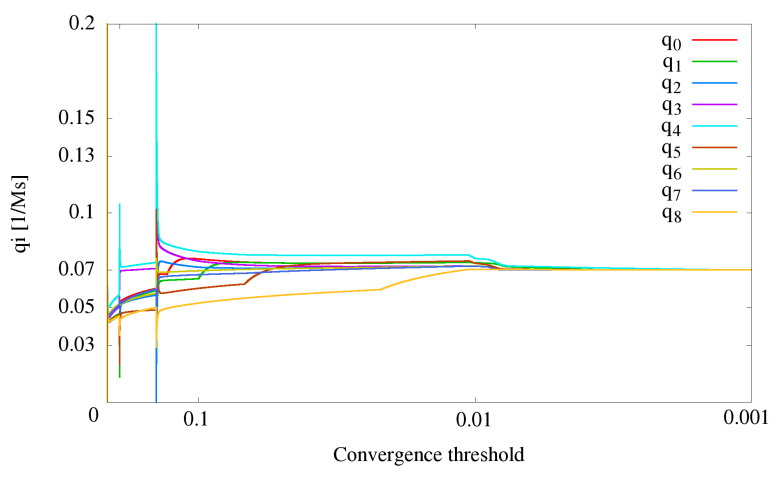

Figure 8: Auxiliary variables for the first topology

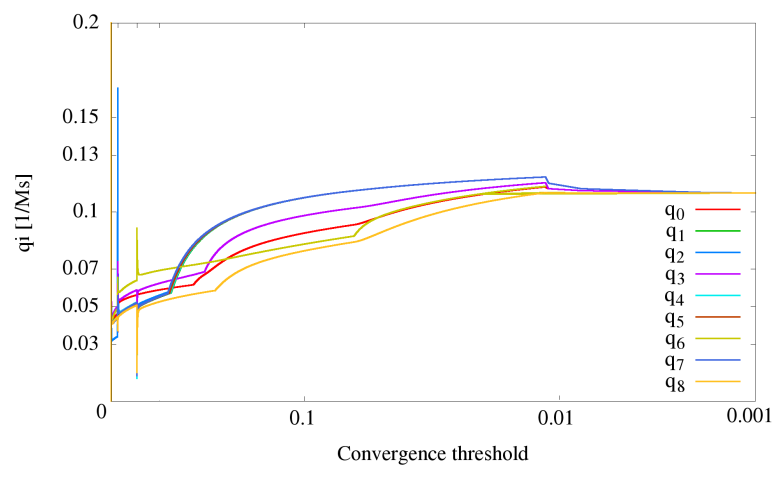

Figure 9: Auxiliary variables for the second topology

Before going further, let us discuss the choice of the sufficient number of paths and how it works. Since both experimentation on the two different topologies lead to the same conclusion, the focus will only be put results of the first topology (T1). Table 3 shows two cases: the

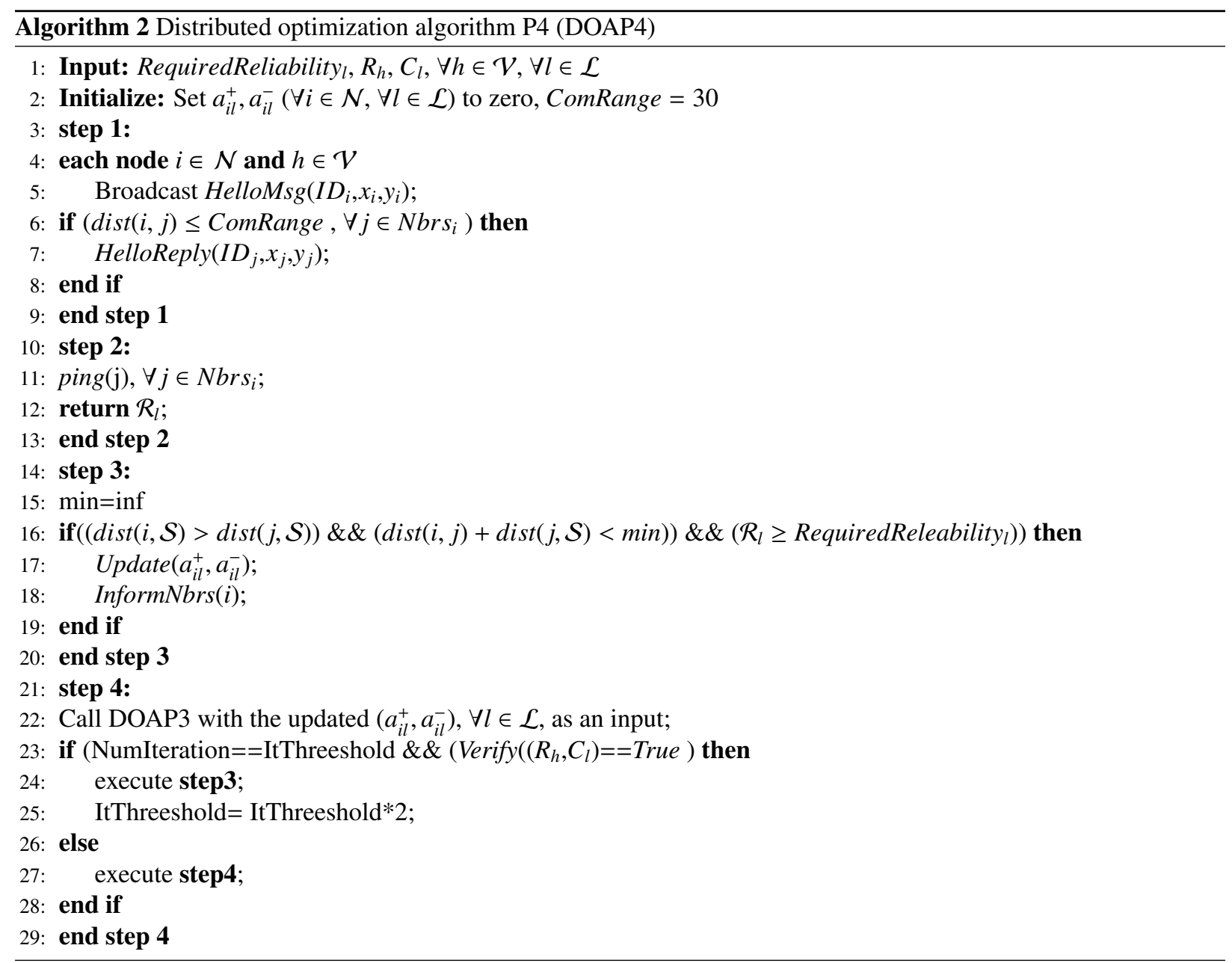



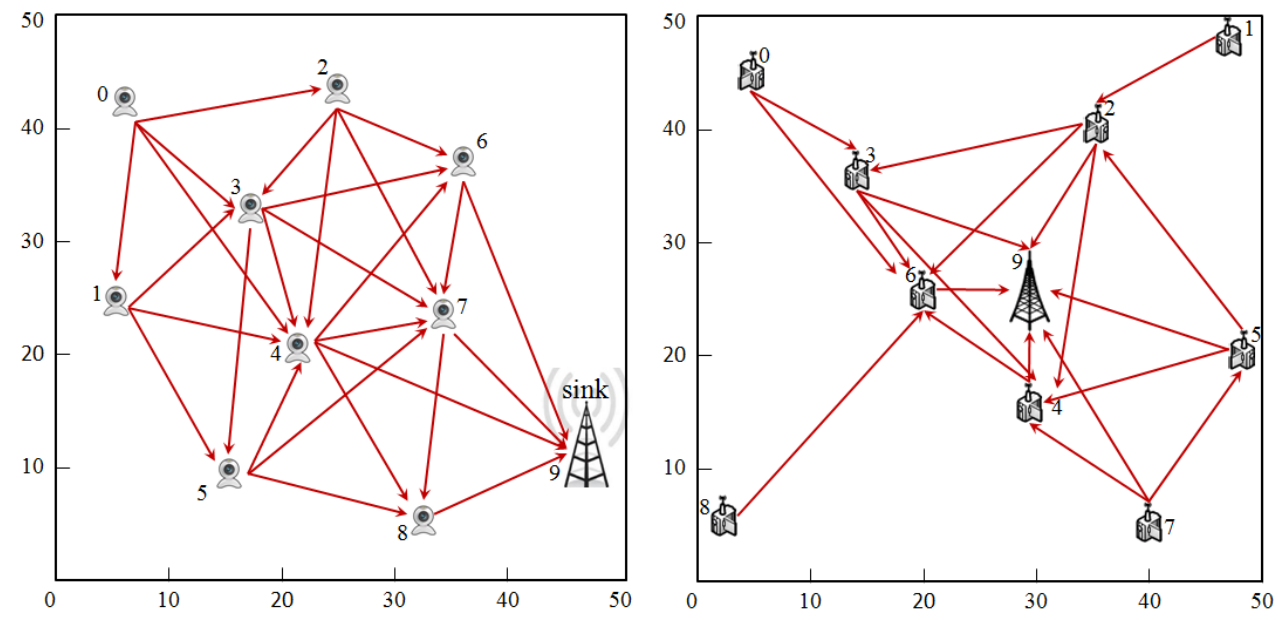

Figure 7: Network topologies: T1 and T2, respectively.

first one, when the node needs to have 3 paths, while in the second case, the node needs to have only 2 paths in order to achieve the performance demands of the target application.

\begin{tabular}{|c|c|c|c|c|}
\hline Nodes & paths & $R_{h} \operatorname{Mbps}($ lst.pr) & $C_{l} \operatorname{Mbps}(2 n d . p r)$ & sufficiency \\
\hline \multirow{6}{*}{0} & 1 path & $R_{0}=0.191541$ & $C_{0}=0.0736882$ & not suff \\
\hline & \multirow{2}{*}{2 paths } & \multirow{2}{*}{$R_{0}=0.180875$} & $C_{0}=0.0855795$ & \multirow{2}{*}{ not suff } \\
\hline & & & $C_{1}=0.0800058$ & \\
\hline & \multirow{3}{*}{3 paths } & \multirow{3}{*}{$R_{0}=0.17962$} & $C_{0}=0.0644174$ & \multirow{3}{*}{ suff } \\
\hline & & & $C_{1}=0.0563247$ & \\
\hline & & & $C_{2}=0.0645586$ & \\
\hline \multirow{4}{*}{5} & 1 path & $R_{5}=0.193454$ & $C_{5}=0.0766058$ & not suff \\
\hline & & & & \\
\hline & \multirow{2}{*}{2 paths } & \multirow{2}{*}{$R_{5}=0.179622$} & $C_{1} 5=0.0980251$ & \multirow{2}{*}{ suff } \\
\hline & & & $C_{1} 6=0.0873981$ & \\
\hline
\end{tabular}

Table 3: Link's capacities Vs link's rates

From the table above, we can observe that the data rate $R_{0}$ is much bigger than the link capacity $C_{0}$ that connects node 0 to its first best neighbor node. To cope with this problem, node 0 chooses its second best downstream node (connected by link $l=1$, in this experimentation). However, both paths seem to be insufficient for the data transmission. Thus, node 0 has to select another downstream node which is considered as the third best neighbor node, instead of node 5 where the selection of only two paths seems to be the adequate number of paths to be used (i.e., $R_{5}<C_{15}+C_{16}$ ).

The main advantage of such a downstream node(s) selection is the progressive path discovery, making it possible to choose not only the best path(s) toward the sink node, but also, to select the sufficient number in order to preserve the energy of each node and thus extend the network lifetime.

\subsection{Optimization cost}

In this subsection we analyze the cost of the optimization steps in terms of energy and duration at each node for the first and second topologies.

\subsubsection{Energy cost}

Figure 10 and Figure 11 depict the percentage of battery consumption of each video sensor node, for the first and second topology, respectively. The latter concerns the total energy requirement for the optimization steps of both P3 and P4. It can be observed that the energy consumed by the highest consumer node in term of battery, in order to ensure the convergence, did not exceed $0.025 \%$ and $0.08 \%$ for the first and second topology, respectively. Thus, it can be concluded that the optimization steps including both $\mathbf{P 3}$ and $\mathbf{P 4}$ consume a negligible amount of energy.

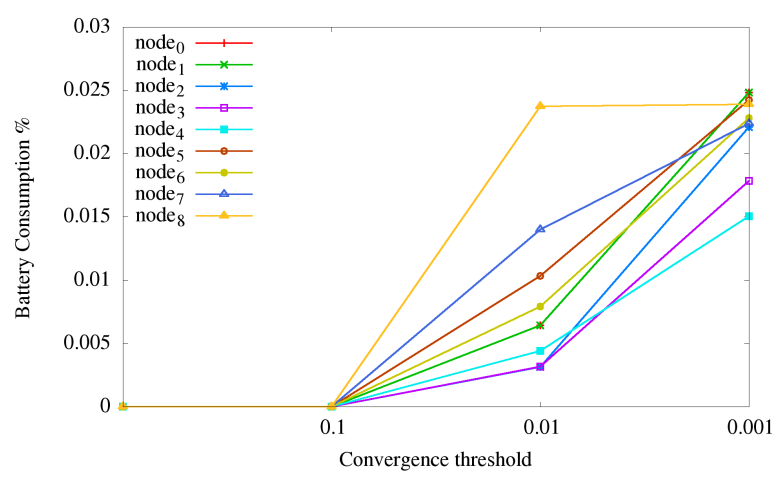

Figure 10: battery consumption of optimization steps for the first topology 


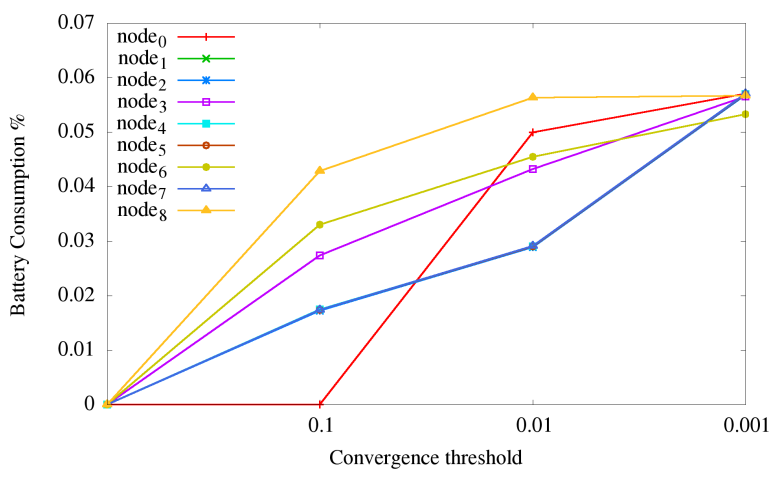

Figure 11: battery consumption of optimization steps for the second topology

\subsubsection{Optimization steps duration}

Let us now evaluate the duration (in minutes) of the optimization steps needed for the system convergence. Figure 12 (first topology) and Figure 13 (second topology) present the optimization steps duration including both $\mathbf{P 3}$ and $\mathbf{P 4}$, for each threshold $T \leq 10^{-3}$. It can be observed that the optimization duration differs from one topology to another, here the optimization duration of the second topology exceeds the optimization duration of the first one. This difference can be explained by the adequate number of paths selected by each video node. Note that, the smaller the number of the selected paths is, the bigger the number of iterations required for the system to converge is, and thus, the longer the optimization duration last.

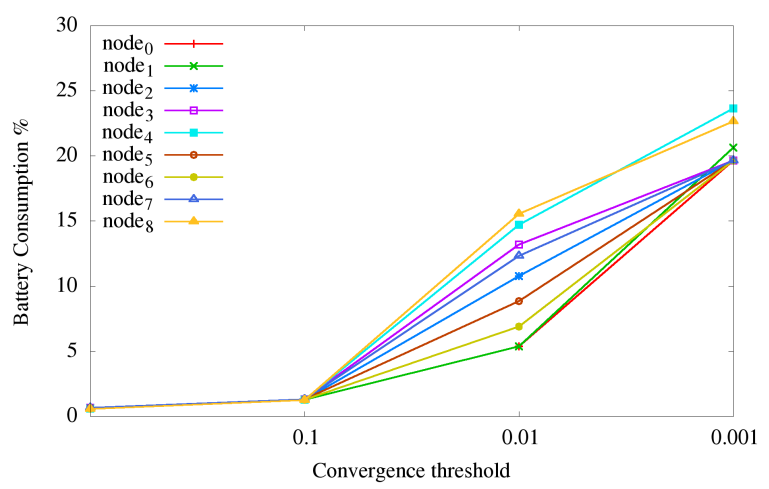

Figure 12: Optimization duration for the first topology

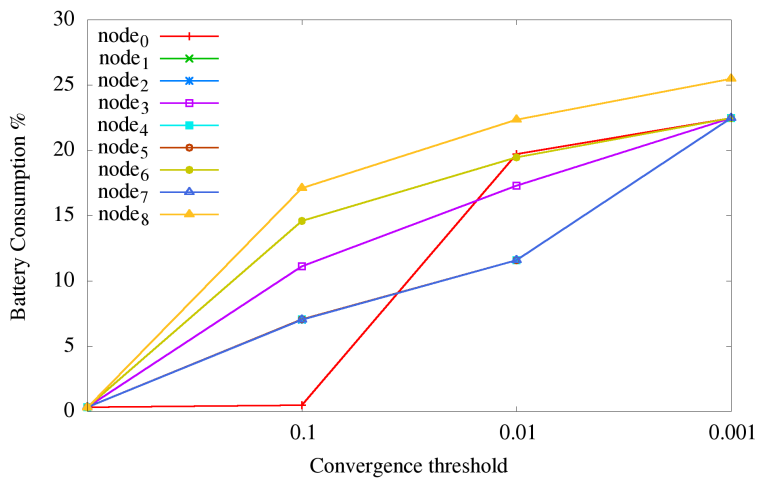

Figure 13: Optimization duration for the second topology

\subsection{Node lifetime improvement}

Let us recall that, the node lifetime is calculated as follows: $T_{i}=B_{i} / P_{i}$.

An this stage of the evaluation, we focus on the remaining lifetime of each video node after each convergence threshold. Taking into account that the network lifetime is defined by the node that has the lowest lifetime, Figure 14, and Figure 15 show the improvement of nodes lifetime for the first and second topology, respectively. It can be observed that the network lifetime has been increased by increasing the lowest node lifetime (namely. node 3 for the first topology and node 0 for the second topology) by at least 7.47 times for the first topology and by 7.67 times for the second one.

This improvement demonstrates the efficiency of the integration of the routing into the analytical model, while respecting the reliability of each path and selecting the sufficient number of paths in order to prolong the network lifetime and respect the desired video quality at the destination level.

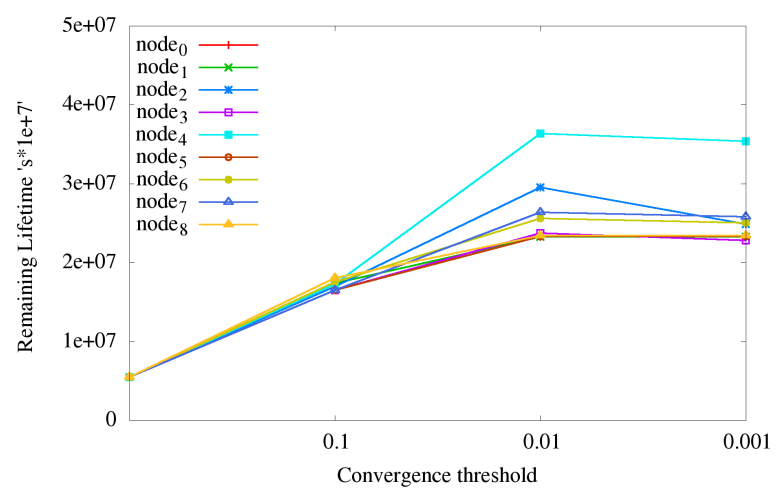

Figure 14: Remaining lifetime after each convergence threshold for the first topology 


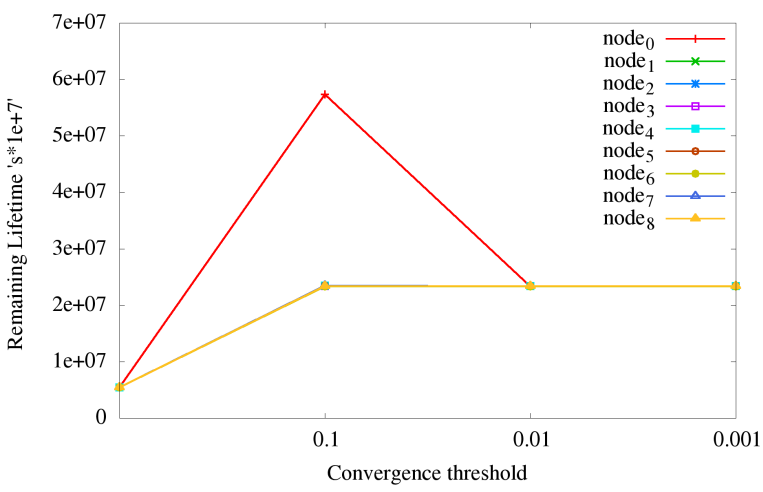

Figure 15: Remaining lifetime after each convergence threshold for the second topology

\section{Conclusion}

In wireless video sensor networks, the video coding and multimedia content delivery are the most energy consuming tasks, and hence impact the overall network lifetime. Thus, both the processing and the delivery of multimedia content should be considered, since their interaction has a major impact on the network lifetime.

In this paper, based on our previous work on power/rate tradeoff for network lifetime maximization, a new analytical model for video coding and multimedia content delivery have been proposed. The optimization problem was solved over a two level optimization, through which each video sensor node chooses the best downstream node(s) based on: the link reliability, the data rate of the video node and the capacity of each link. At the same time, the trade-off between the encoding power at the source level and the desired video quality at the destination level is ensured.

Simulation results prove the efficiency of the proposed solution, given its minimal requirements in terms of computational power and energy consumption, while ensuring the desired video quality at the destination level to be transmitted to the cloud for a relevant analysis.

\section{REFERENCES}

[1] C. Ingraham, Wonkblog the astonishing human potential wasted on commutes, https://www. washingtonpost.com/news/wonk/wp/2016/02/25/ how-much-of-your-life-youre-wasting-on-your/ /-commute/?utm_term=.647aaa2e1856

[2] N. N.V., Moscow installs smart parking system sensit, http://www.nedapidentification.com/news/news/ moscow-installs-sensit (2017).

[3] I. T. Almalkawi, M. Guerrero Zapata, J. N. Al-Karaki, J. Morillo-Pozo, Wireless multimedia sensor networks: current trends and future directions, Sensors 10 (7) (2010) 6662-6717.
[4] N. Khernane, J.-F. Couchot, A. Mostefaoui, Routing impact on network lifetime maximization using power/rate trade-off in wvsn, in: The 13th International Wireless Communications and Mobile Computing Conference, IEEE, 2017.

[5] N. Khernane, J.-F. Couchot, A. Mostefaoui, Maximizing network lifetime in wireless video sensor networks under quality constraints, in: Proceedings of the 14th ACM International Symposium on Mobility Management and Wireless Access, ACM, 2016, pp. 59-66.

[6] Z. He, D. Wu, Resource allocation and performance analysis of wireless video sensors, Electrical and Computer Engineering publications (MU), 2006, vol. 16, no 5, p. 590-599.

[7] Y. Lin, E. Gürses, A. N. Kim, A. Perkis, Optimal joint powerrate adaptation for error resilient video coding, in: Electronic Imaging 2008, International Society for Optics and Photonics, 2008, pp. 68220Q-68220Q

[8] Y. He, I. Lee, L. Guan, Distributed algorithms for network lifetime maximization in wireless visual sensor networks, Circuits and Systems for Video Technology, IEEE Transactions on 19 (5) (2009) 704-718

[9] A. Arar, A. Mohamed, A. A. El-Sherif, V. C. Leung, Optimal resource allocation for green and clustered video sensor networks, IEEE Systems Journal.

[10] E. Gürses, Y. Lin, R. Boutaba, Distributed quality-lifetime maximization in wireless video sensor networks, in: Communications, 2009. ICC'09. IEEE International Conference on, IEEE, 2009, pp. 1-6.

[11] H. Nama, M. Chiang, N. Mandayam, Optimal utility-lifetime trade-off in self-regulating wireless sensor networks: a distributed approach, in: Information Sciences and Systems, 2006 40th Annual Conference on, IEEE, 2006, pp. 789-794.

[12] D. P. Bertsekas, J. N. Tsitsiklis, Parallel and distributed computation: numerical methods, Vol. 23, Prentice hall Englewood Cliffs, NJ, 1989.

[13] J. Zou, H. Xiong, C. Li, R. Zhang, Z. He, Lifetime and distortion optimization with joint source/channel rate adaptation and network coding-based error control in wireless video sensor networks, IEEE Transactions on Vehicular Technology 60 (3) (2011) 1182-1194.

[14] J. Zou, C. Tan, R. Zhang, H. Xiong, Modeling and optimization of network lifetime in wireless video sensor networks, in: Communications (ICC), 2010 IEEE International Conference on, IEEE, 2010, pp. 1-6.

[15] A. Arar, A. Mohamed, A. A. El-Sherif, V. C. Leung, Optimal resource allocation for green and clustered video sensor networks, IEEE Systems Journal, 2017.

[16] L. Zhou, D. Wu, Z. Dong, X. Li, When collaboration hugs intelligence: Content delivery over ultra-dense networks, IEEE Communications Magazine 55 (12) (2017) 91-95.

[17] D. Wu, L. Zhou, Y. Cai, Social-aware rate based content sharing mode selection for $\mathrm{d} 2 \mathrm{~d}$ content sharing scenarios, IEEE Transactions on Multimedia 19 (11) (2017) 2571-2582.

[18] J. Park, S. Sahni, Maximum lifetime routing in wireless sensor networks, Computer \& Information Science \& Engineering, University of Florida.

[19] R. Madan, S. Lall, Distributed algorithms for maximum lifetime routing in wireless sensor networks, IEEE Transactions on wireless communications 5 (8) (2006) 2185-2193.

[20] M. Tahir, R. Farrell, Optimal communication-computation tradeoff for wireless multimedia sensor network lifetime maximization, in: Wireless Communications and Networking Conference, 2009. WCNC 2009. IEEE, IEEE, 2009, pp. 1-6.

[21] L. Cobo, A. Quintero, S. Pierre, Ant-based routing for wireless multimedia sensor networks using multiple qos metrics, Computer networks 54 (17) (2010) 2991-3010. 
[22] M. A. Razzaque, C. S. Hong, S. Lee, Data-centric multiobjective qos-aware routing protocol for body sensor networks, Sensors 11 (1) (2011) 917-937.

[23] S. Chen, Z. Yuan, G.-M. Muntean, An energy-aware routing algorithm for quality-oriented wireless video delivery, IEEE Transactions on Broadcasting 62 (1) (2016) 55-68.

[24] H.-W. Kim, A. Kachroo, Low power routing and channel allocation of wireless video sensor networks using wireless link utilization, Ad Hoc \& Sensor Wireless Networks 30 (1-2) (2016) 83-112.

[25] L. Wang, Q. Xu, Gps-free localization algorithm for wireless sensor networks, Sensors 10 (6) (2010) 5899-5926.

[26] L. Benoit, P. Briole, O. Martin, C. Thom, J.-P. Malet, P. Ulrich, Monitoring landslide displacements with the geocube wireless network of low-cost gps, Engineering Geology 195 (2015) 111121.

[27] X. Wu, G. Ding, B. Bhargava, S. Lei, Improving throughput by link distance control in a multi-rate ad hoc network.

[28] T. S. Rappaport, et al., Wireless communications: principles and practice, Vol. 2, Prentice Hall PTR New Jersey, 1996.

[29] M. Cheng, X. Gong, L. Cai, Joint routing and link rate allocation under bandwidth and energy constraints in sensor networks, IEEE Transactions on Wireless Communications 8 (7).

[30] M. X. Cheng, L. Yin, Energy-efficient data gathering algorithm in sensor networks with partial aggregation, International Journal of Sensor Networks 4 (1-2) (2008) 48-54.

[31] D. P. Bertsekas, R. G. Gallager, Data networks, Vol. 2, pages 363-491. Prentice-Hall, Inc., 1987

[32] D. Seo, M. Thottethodi, Disjoint-path routing: Efficient communication for streaming applications, in: Parallel \& Distributed Processing, 2009. IPDPS 2009. IEEE International Symposium on, IEEE, 2009, pp. 1-12

[33] F. Iqbal, F. A. Kuipers, Disjoint paths in networks, Wiley Encyclopedia of Electrical and Electronics Engineering.

[34] D. P. Palomar, M. Chiang, A tutorial on decomposition methods for network utility maximization, Selected Areas in Communications, IEEE Journal on 24 (8) (2006) 1439-1451.

[35] D. P. Bertsekas, A. E. Ozdaglar, A. Nedic, Convex analysis and optimization Athena scientific optimization and computation series, Athena Scientific, Belmont (Mass.), 2003. URL http://opac .inria.fr/record=b1108032

[36] A. Varga, et al., The omnet++ discrete event simulation system, in: Proceedings of the European simulation multiconference (ESM'2001), Vol. 9, S 185, sn, 2001, p. 65.

[37] A. Köpke, M. Swigulski, K. Wessel, D. Willkomm, P. Haneveld, T. E. Parker, O. W. Visser, H. S. Lichte, S. Valentin, Simulating wireless and mobile networks in omnet ++ the mixim vision, in: Proceedings of the 1st international conference on Simulation tools and techniques for communications, networks and systems \& workshops, ICST (Institute for Computer Sciences, SocialInformatics and Telecommunications Engineering), 2008, p. 71

[38] P. Chen, P. Ahammad, C. Boyer, S.-I. Huang, L. Lin, E. Lobaton, M. Meingast, S. Oh, S. Wang, P. Yan, et al., Citric: A lowbandwidth wireless camera network platform, in: Distributed smart cameras, 2008. ICDSC 2008. Second ACM/IEEE international conference on, IEEE, 2008, pp. 1-10.

[39] W.-c. Feng, E. Kaiser, W. C. Feng, M. L. Baillif, Panoptes: scalable low-power video sensor networking technologies, ACM Transactions on Multimedia Computing, Communications, and Applications (TOMM) 1 (2) (2005) 151-167.

[40] J. Boice, X. Lu, C. Margi, G. Stanek, G. Zhang, R. Manduchi, K. Obraczka, Meerkats: A power-aware, self-managing wireless camera network for wide area monitoring, in: Proc. Workshop on Distributed Smart Cameras, Citeseer, 2006, pp. 393-422.

[41] N. Khernane, J.-F. Couchot, A. Mostefaoui, Optimal power/rate trade-off for internet of multimedia things lifetime maximization under dynamic links capacity, Future Generation Computer Systems, Elsevier, 2017.

[42] D. Tse, P. Viswanath, Fundamentals of wireless communication, Cambridge university press, 2005

Nesrine Khernane was born in

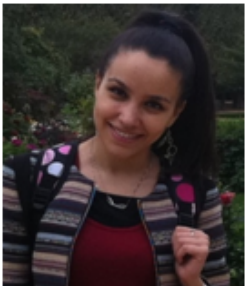
Algeria (1990). She received her Master degree in Smart Mobility, Internet of things (SMIT) from the Piere et Marie Curie University (Sorbonne University) in 2015. She is currently a $\mathrm{PhD}$ student at the university of Bourgogne France Comte (France), under the direction of Ahmed Mostefaoui and Jean-François Couchot. Her main areas of research interests cover the routing, security and privacy in Wireless Multimedia Sensor Networks and Wireless Body area networks.

Jean-François COUCHOT is

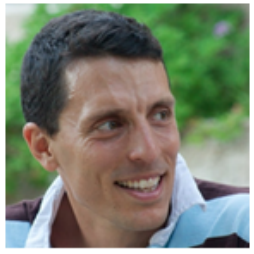
an Assistant Professor of the FEMTO-ST Institute (CNRS) at Univ of Bourgogne FrancheComté. He received a Ph.D. in Computer Science in 2006 in the FEMTO-ST Institute. He has applied for a postdoctoral position at INRIA Saclay Île de France in 2006. His research focuses on discrete dynamic systems (with applications into data hiding, watermarking, pseudorandom number generators, hash function) and on bioinformatic, especially in gene evolution prediction. He has written more than 40 scientific articles in these areas.

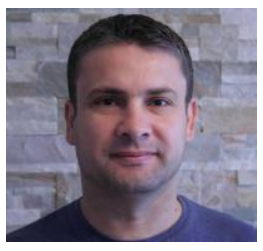

Ahmed Mostefaoui is currently an associate professor at the University of Bourgogne Franche Comte, France, since 2000. He received the M.S. and Ph.D. degrees in computer science from Ecole Normale Suprieure de Lyon (France) in 1996 and 2000, respectively. His research interests are in distributed algorithms in wireless ad-hoc and sensor networks emphasizing both practical and theoretical issues, multimedia systems and networking, in particular, distributed architectures. mathematics. 